\title{
X-ray flux variability of active galactic nuclei observed using NuSTAR
}

\author{
Priyanka Rani, ${ }^{\star}$ C. S. Stalin and Suvendu Rakshit \\ Indian Institute of Astrophysics, Koramangala, Bangalore 560034, India
}

Accepted 2016 December 08. Received 2016 December 08; in original form 2016 September 26

\begin{abstract}
We present results on a systematic study of flux variability on hourly time scales in a large sample of active galactic nuclei (AGN) in the 3-79 $\mathrm{keV}$ band using data from NuSTAR. Our sample consists of 4 BL Lac objects (BL Lacs), 3 flat spectrum radio quasars (FSRQs) 24 Seyfert 1, 42 Seyfert 2 and 8 narrow line Seyfert 1 (NLSy1) galaxies. We find that in the $3-79 \mathrm{keV}$ band, about $65 \%$ of the sources in our sample show significant variations on hourly time scales. Using Mann-Whitney U-test and Kolmogorov-Smirnov test, we find no difference in the variability behaviour between Seyfert 1 and 2 galaxies. The blazar sources (FSRQs and BL Lacs) in our sample, are more variable than Seyfert galaxies that include Seyfert 1 and Seyfert 2 in the soft $(3-10 \mathrm{keV})$, hard $(10-79 \mathrm{keV})$ and total $(3-79 \mathrm{keV})$ bands. NLSy1 galaxies show the highest duty cycle of variability (87\%), followed by BL Lacs $(82 \%)$, Seyfert galaxies $(56 \%)$ and FSRQs $(23 \%)$. We obtained flux doubling/halving time in the hard X-ray band less than 10 min in 13 sources. For PKS 2155-304, we find the shortest flux doubling time of $1.65 \pm 0.16$ min the shortest known in the hard X-ray band from any blazar. The flux variations between the hard and soft bands in all the sources in our sample are consistent with zero lag.
\end{abstract}

Key words: galaxies: active-X-rays: galaxies.

\section{INTRODUCTION}

There is convincing evidence that accretion onto supermassive black holes powers active galactic nuclei (AGN; Rees 1984). A vast majority of about $85 \%$ of them emit little or no radio emission, these AGN are termed radio-quiet AGN and a minority of about $15 \%$ called radio-loud AGN have large scale relativistic jets and emit copiously in the radio band. It is still not known what triggers relativistic jets in only a small fraction of AGN and thereby giving rise to the apparent radio-loud radio-quiet dichotomy (Ivezić et al. 2002; Cirasuolo et al. 2003). Both radio-loud and radio-quiet AGN may be categorized into several sub-classes such as Seyfert galaxies (Seyfert 1 and 2), radio galaxies, blazars comprising flat spectrum radio quasars (FSRQs) and BL Lac objects (BL Lacs) etc., According to the unification model, the observed differences between the different types of AGN are in part due to orientation effects (Antonucci 1993; Urry \& Padovani 1995). One of the defining characteristics of AGN is that they show flux variations across the entire accessible electromagnetic spectrum (Wagner \& Witzel 1995; Ulrich

^ E-mail: priyanka@iiap.res.in et al. 1997) over a wide range of timescales ranging from minutes to months and years. Though flux variations in AGN are known since their discovery, we still do not completely understand the physical processes that cause such variations (McHardy et al. 2006). Also, it is not unambiguously known if the same physical processes are responsible for the observed flux variations in different types of AGN. In spite of our lack of a clear knowledge on the cause of flux variations, this characteristic of AGN when probed particularly in the $\mathrm{X}$-ray band can give important constraints on the physical properties in the innermost regions of AGN and can even provide clues on the observed radio-loud and radio-quiet dichotomy (Gliozzi et al. 2002).

Among the X-ray band, hard X-ray (with energies greater than $15 \mathrm{keV}$ ) variability in particular can provide clues about the physics of the central regions of AGN and kinematics of the jet as it is less affected by absorption than soft X-rays, when the line of sight hydrogen column density is less than $10^{23} \mathrm{~cm}^{-2}$ (Soldi et al. 2014). Hard X-rays can thus serve as an effective wavelength range to probe the intrinsic properties of the different classes of AGN. Therefore, the study of hard X-ray properties of the various types of AGN in general and hard X-ray flux variability in par- 
ticular can be used to test the validity of the unification model of AGN (Beckmann \& Shrader 2012; Ghisellini et al. 1994; Urry \& Padovani 1995). Various models are available in the literature on the physical processes that cause hard X-ray emission in AGN. According to Haardt \& Maraschi (1993), the hard X-ray emission in radio-quiet AGN is due to the Comptonization of the soft accretion disk photons by a plasma of hot electrons situated above the disk. In the case of radio-loud AGN, in addition to the process that produces hard X-ray emission in radio-quiet AGN, there are additional contributions through inverse Compton (IC) processes from relativistic electrons in the jet.

In any given X-ray band, the observed characteristics of different types of AGN depend on the physical processes that contribute to the observed spectral energy distribution (SED) in that band. In blazars, the broad band SED shows two broad emission peaks: the low energy peak is produced by synchrotron emission and the high energy peak is produced by IC emission process. The seed photons for IC can be from various sources such as the synchrotron photons themselves (Konigl 1981), accretion disk (Dermer \& Schlickeiser 1993; Boettcher et al. 1997), the broad line region (BLR, Sikora et al. 1994; Ghisellini \& Madau 1996) or the torus (Błażejowski et al. 2000). These external photon fields contribute differently in individual blazars and this can explain the varied nature of the high energy component in the SED of blazars. Depending on the location of the synchrotron peak in their SEDs, blazars are further subdivided (Padovani \& Giommi 1995; Abdo et al. 2010) into high synchroton peaked blazars (HSP, with synchrotron emission peaking at X-ray energies with $\nu_{s}>10^{15} \mathrm{~Hz}$ ), intermediate synchrotron peaked blazars (ISP, $10^{14}<\nu_{s}<10^{15} \mathrm{~Hz}$ and low synchrotron peaked blazars (LSP, with synchrotron emission peaking in the IR; $\nu_{s}<10^{14} \mathrm{~Hz}$ ). In HSPs, the Xray spectrum falls in the synchrotron region, thereby showing large amplitude X-ray variations at long as well as short time scales (Sembay et al. 1993; Tanihata et al. 2000; Zhang et al. 2002, 2005), however, less variable in the optical (Heidt 1996). On the other hand, LSPs are more variable in the optical (Heidt 1996) compared to the X-ray (Gupta et al. 2016) band which falls in the IC region of their SED. Nonetheless, it is not always the case and there are instances where the $\mathrm{X}$-ray spectrum is found to have contribution both from the high energy tail of the synchrotron component and the IC component. This has been noticed in several LSP blazars (Tagliaferri et al. 2000; Tanihata et al. 2003; Wierzcholska \& Wagner 2016) and in one HSP blazar (Kataoka \& Stawarz 2016) namely Mrk 421.

Blazars are known to show fast variability. Detection of such fast variability time scales can set an upper limit on the size of the emission region as $R_{s}<\mathrm{c} \delta \mathrm{t}_{\text {var }}$, via light travel time arguments. Here, $\delta$ is the Doppler factor, and $t_{v a r}$ is the variability time scale. Such fast time scale of variations (in the order of minutes) characterised by the flux doubling/halving time scale are often seen in high energy $\gamma$-rays (Aharonian et al. 2007; Gaidos et al. 1996). Also, in HSP blazars correlated X-ray and $\gamma$-ray variations are found (Baloković et al. 2016; Aleksić et al. 2015) which is very well expected in the one zone leptoinc model of blazar emission, wherein, both hard X-rays and $\gamma$-rays are produced by the same population of relativistic electrons in the jet. Detection of such short time scale X-ray flares with/without a high en- ergy $\gamma$-ray counterpart will constrain the radiative processes operating in the sub-parsec scales of AGN. Detection of very small flux doubling/halving time scale is thus an important addition to the knowledge of AGN flux variability. Previous efforts to search for flux doubling/halving time scale $<15$ minutes in the X-ray band were negative (Pryal et al. 2015). Therefore, any evidences for the presence of minute scale flux doubling/halving time scale is more important.

In the soft X-ray band, with X-rays having energies < $10 \mathrm{keV}$, numerous results on the flux variability nature of AGN on time scales ranging from hours to months and years are available mainly based on observations from Rossi X-ray Timing Explorer (RXTE) and XMM-Newton (Nandra et al. 1997; Fiore et al. 1998; Turner et al. 1999; Uttley et al. 2002; Markowitz et al. 2003; Soldi et al. 2008; McHardy 2010). On the other hand, studies on the hard X-ray variability of AGN are very limited. They include hard X-ray spectral variability based on observations from BeppoSAX (Petrucci et al. 2000), INTEGRAL (Petrucci et al. 2013) and Suzaku (Reis et al. 2012). Long term hard X-ray monitoring observations have been recently possible owing to the observations by the Burst Alert Telescope (BAT; Barthelmy et al. 2005) instrument on board the Swift satellite (Gehrels et al. 2004). Because of Swift/BAT's observing capability in survey mode and its large field of view of $\sim 1.4 \mathrm{sr}$, we have long term monitoring data on a large sample of bright AGN from BAT. Using this data set, AGN have been studied for long term variability on time scale of days to years (Gehrels et al. 2004; Caballero-Garcia et al. 2012; Soldi et al. 2014). However, studies on the hard X-ray variations on time scales of the order of hours are available only on few individual objects such as Mrk 421 (Paliya et al. 2015) using the focusing hard X-ray telescope Nuclear Spectroscopic Telescope Array (NuSTAR; Harrison et al. 2013), sources such as BL Lac (Ravasio et al. 2003), ON 231 (Tagliaferri et al. 2000), Mrk 421 (Maraschi et al. 1999), Mrk 501 (Pian et al. 1998) etc. from BeppoSAX as well as observations from RXTE (Fossati et al. 2008). These observations pertaining to sources in different brightness states indicate different variability behaviour between hard and soft bands, which point to different physical processes contributing to the soft and hard $\mathrm{X}$-ray emission.

Observations using NuSTAR with its high sensitivity and wide spectral coverage between $3-79 \mathrm{keV}$ is ideal to constrain the X-ray emission processes that contribute to the X-ray emission over a wide spectral range in AGN. We investigate here for the first time the hard X-ray flux variations on hourly time scales in the largest sample of AGN that includes both radio-loud and radio-quiet sources. The main motivation here is to understand (i) the similarities and differences in the flux variability nature of different types of AGN over a wide waveband of $3-79 \mathrm{keV}$, (ii) the differences if any between flux variations in soft and hard X-ray bands and (iii) the presence of fast variations with flux doubling/halving time scale of few minutes. For this we use the publicly available data from observations by NuSTAR. The structure of the paper is as follows. In section 2, we describe the sample used and data reduction. In Section 3 we describe the various analysis carried out on the data set. We discuss our results in Section 4 and summarize the results in Section 5. We adopt a cosmology with $H_{0}=71 \mathrm{~km} \mathrm{~s}^{-1} \mathrm{Mpc}^{-1}$, $\Omega_{\Lambda}=0.73$ and $\Omega_{m}=0.27$. 


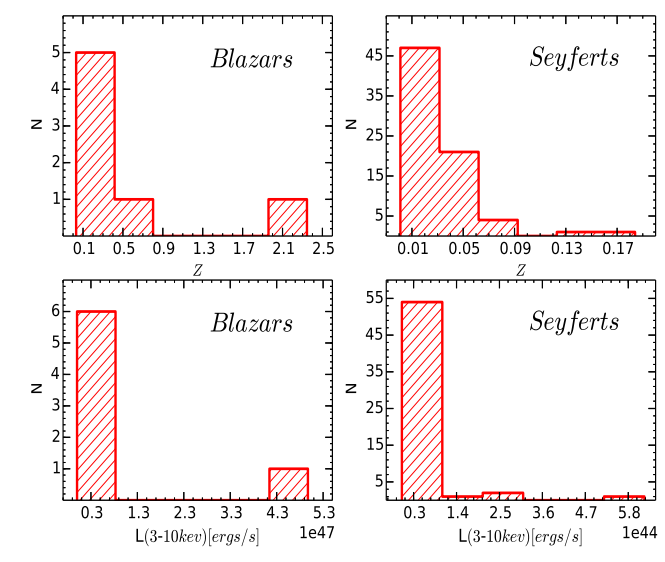

Figure 1. The distribution of redshift and the $3-10 \mathrm{keV}$ luminosity of the sources. The left hand panels are for blazars, that includes FSRQs and BL Lacs, and the right hand panel are for Seyferts comprising Seyfert 1 and 2 galaxies.

\section{SAMPLE AND DATA REDUCTION}

The goal of this work is to find the hard X-ray flux variations in different types of AGN. For this we have used the data from NuSTAR. NuSTAR (Harrison et al. 2013) launched in June 2012, is the first focusing hard X-ray telescope. It has a field of view $13^{\prime} \times 13^{\prime}$. It consists of two co-aligned Xray detector pairs with corresponding focal plane modules FPMA and FPMB ${ }^{1}$. NuSTAR observations of all AGN that have become public before March 2015 have been used in this work. Our sample thus consists of 81 AGN, among these 4 are BL Lacs, 3 are FSRQs, 24 are Seyfert 1 galaxies, 42 are Seyfert 2 galaxies and 8 are narrow line Seyfert 1 (NLSy1) galaxies. The details of the sources used in this study are given in Table 1 . The distribution of redshifts and the $3-10$ $\mathrm{keV}$ luminosity of the sources studied are shown in Figure 1 separately for radio-quiet sources and blazars.

Reduction of the NuSTAR data was done using the data analysis software NuSTARDAS v.1.4.1 distributed by the High Energy Astrophysics Archive Research Center (HEASARC). The calibrated, cleaned and screened event files were generated using the nupipeline task and using CALDB 20141107. A circular region of $60^{\prime \prime}$ radius was taken to extract the source and background counts on the same detector. We extracted the 5 min binned light curves in the energy range of 3-79 $\mathrm{keV}$ in each focal plane module FPMA and FPMB using the nuproducts package available in $N u S$ TARDAS. Further, these light curves were divided into two bands: $3-10 \mathrm{keV}$ and $10-79 \mathrm{keV}$. To generate light curves, the count rates of the two modules FPMA and FPMB are combined using 'lcmath' task included in FTOOLS V.4.0. Sample light curves of the BL Lac object Mrk 421 are shown in Figure 2.

\footnotetext{
1 https://heasarc.gsfc.nasa.gov/docs/nustar/
}

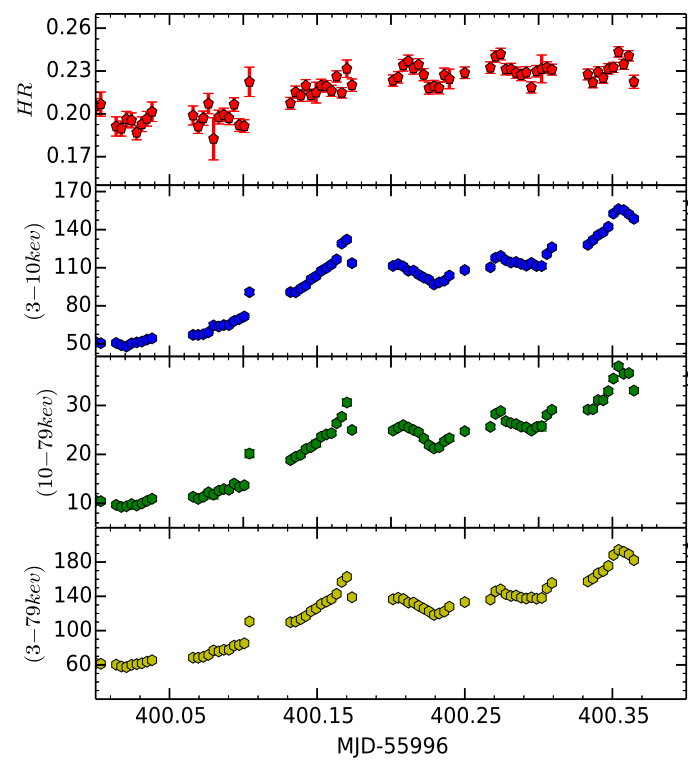

Figure 2. Light curves of the BL Lac object Mrk 421 corresponding to the observational ID 60002023031 and observed on 201304-14 for a duration of $15606 \mathrm{sec}$. From the top to the bottom are shown the HR variation, flux variations in the energy ranges of 3-10 keV (soft band), 10-79 keV (hard band) and 3-79 keV (total band) respectively. Each point corresponds to a binning of 300 seconds and the fluxes are in units of counts $\mathrm{s}^{-1}$.

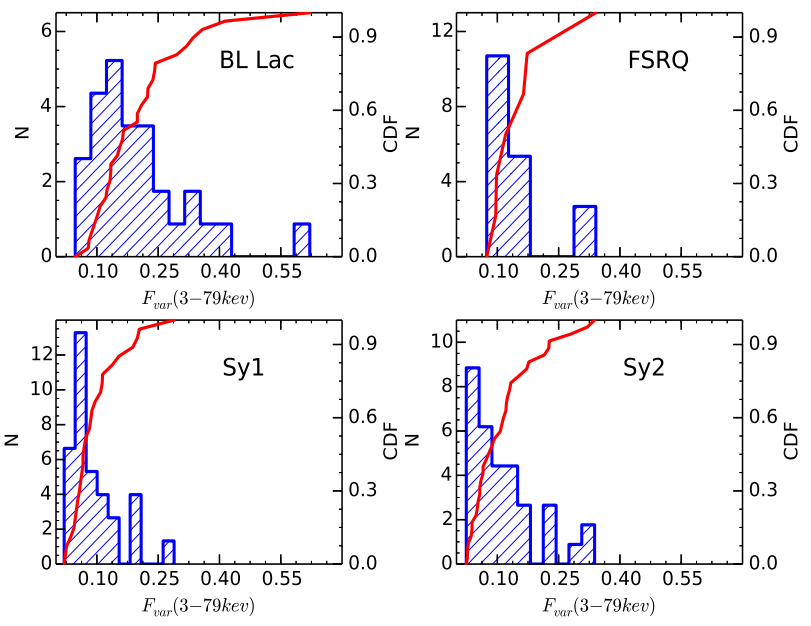

Figure 3. Histogram and cumulative distribution function (CDF) of $F_{\text {var }}$ in the 3-79 keV band for different classes of AGN. The values of $\mathrm{CDF}$ are given in the right of each figure.

\section{ANALYSIS}

\subsection{Variability Amplitude}

To characterize the flux variations we used the fractional root mean square (rms) variability amplitude $F_{\text {var }}$ (Edelson et al. 2002; Vaughan et al. 2003). This gives an estimate of the intrinsic variability amplitude relative to the mean count 
Table 1. Information on the sources studied for flux variability. The quoted magnitudes are the V-band magnitudes taken from VéronCetty \& Véron (2010) except for those appended with a * for which it is from NED (http://ned.ipac.caltech.edu/).

\begin{tabular}{|c|c|c|c|c|c|c|c|c|c|c|c|}
\hline Name & $\alpha(2000)$ & $\delta(2000)$ & $\begin{array}{l}\mathrm{V} \\
(\mathrm{mag})\end{array}$ & $z$ & Type & Name & $\alpha(2000)$ & $\delta(2000)$ & $\begin{array}{l}\mathrm{V} \\
(\mathrm{mag})\end{array}$ & $z$ & Type \\
\hline Mrk 335 & $00: 06: 19.5$ & $+20: 12: 11$ & 13.85 & 0.026 & Sy1 & $3 \mathrm{C} 273$ & $12: 29: 06.7$ & $+02: 03: 08$ & 12.85 & 0.158 & FSRQ \\
\hline NGC 424 & $01: 11: 27.7$ & $-38: 05: 10$ & 14.12 & 0.011 & Sy2 & 3C 279 & $12: 56: 11.1$ & $-05: 47: 22$ & 17.75 & 0.538 & FSRQ \\
\hline NGC 513 & $01: 24: 26.8$ & $+33: 47: 58$ & 13.40 & 0.019 & Sy2 & Mrk 231 & $12: 56: 14.2$ & $+56: 52: 25$ & 13.84 & 0.041 & Sy1 \\
\hline NGC 612 & $01: 33: 57.8$ & $-36: 29: 36$ & 13.20 & 0.030 & Sy2 & Mrk 248 & $13: 15: 17.2$ & $+44: 24: 26$ & 15.10 & 0.036 & Sy2 \\
\hline $\mathrm{MCG}+01-05-047$ & $01: 52: 49.0$ & $-03: 26: 49$ & 14.24 & 0.017 & Sy2 & MCG $-06-30-15$ & $13: 35: 53.4$ & $-34: 17: 48$ & 13.61 & 0.008 & Sy1 \\
\hline NGC 788 & 02:01:06.5 & $-06: 48: 56$ & 12.76 & 0.013 & Sy2 & NGC 5252 & $13: 38: 15.9$ & $+04: 32: 33$ & 14.21 & 0.022 & Sy2 \\
\hline NGC 985 & $02: 34: 37.8$ & $-08: 47: 15$ & 14.28 & 0.043 & Sy1 & NGC 5273 & $13: 42: 08.3$ & $+35: 39: 15$ & 13.12 & 0.003 & Sy2 \\
\hline NGC 1068 & $02: 42: 40.7$ & $-00: 00: 47$ & 10.83 & 0.003 & Sy2 & Mrk 273 & $13: 44: 42.1$ & $+55: 53: 13$ & 14.91 & 0.037 & Sy2 \\
\hline $1 \mathrm{H} 0323+342$ & $03: 24: 41.2$ & $+34: 10: 45$ & 15.72 & 0.061 & NLSy1 & IC $4329 A$ & $13: 49: 19.3$ & $-30: 18: 34$ & 13.66 & 0.016 & Sy1 \\
\hline NGC 1320 & $03: 24: 48.7$ & $-03: 02: 32$ & 14.00 & 0.009 & Sy2 & PKS 1409-651 & $14: 13: 09.8$ & $-65: 20: 17$ & 12.10 & 0.001 & Sy2 \\
\hline NGC 1365 & $03: 33: 36.4$ & $-36: 08: 24$ & 12.95 & 0.006 & Sy2 & NGC 5506 & $14: 13: 14.8$ & $-03: 12: 26$ & 14.38 & 0.007 & Sy1 \\
\hline $3 \mathrm{C} 120$ & $04: 33: 11.1$ & $+05: 21: 15$ & 15.05 & 0.033 & Sy1 & NGC 5548 & $14: 17: 59.6$ & $+25: 08: 13$ & 13.73 & 0.017 & Sy1 \\
\hline $\mathrm{MCG}+03-13-01$ & $04: 46: 29.7$ & $+18: 27: 40$ & 15.00 & 0.016 & Sy2 & NGC 5674 & $14: 33: 52.3$ & $+05: 27: 30$ & 13.70 & 0.025 & Sy2 \\
\hline IRAS $04507+0358$ & $04: 53: 25.7$ & $+04: 03: 42$ & 15.00 & 0.030 & Sy2 & Mrk 477 & $14: 40: 38.1$ & $+53: 30: 16$ & 15.03 & 0.038 & Sy2 \\
\hline XSS J05054-2348 & 05:05:45.7 & $-23: 51: 14$ & 17.00 & 0.035 & Sy2 & NGC 5728 & $14: 42: 23.9$ & $-17: 15: 11$ & 13.40 & 0.009 & Sy2 \\
\hline ZW 468.002 & 05:08:19.7 & $+17: 21: 47$ & 13.50 & 0.017 & Sy2 & IGR J14552-5133 & $14: 55: 17.8$ & $-51: 34: 17$ & 17.10 & 0.016 & NLSy1 \\
\hline ARK 120 & $05: 16: 11.4$ & $-00: 09: 00$ & 13.92 & 0.033 & Sy1 & IC $4518 \mathrm{~A}$ & $14: 57: 41.2$ & $-43: 07: 56$ & 15.00 & 0.016 & Sy2 \\
\hline IRAS 05189-2524 & 05:21:01.4 & $-25: 21: 45$ & 14.75 & 0.042 & Sy2 & SWIFT J1514.5-8123 & $15: 14: 42.0$ & $-81: 23: 38$ & $* 17.3$ & 0.068 & Sy1 \\
\hline NGC 2110 & $05: 52: 11.4$ & $-07: 27: 23$ & 13.51 & 0.007 & Sy2 & Mrk 290 & $15: 35: 52.3$ & $+57: 54: 09$ & 15.30 & 0.030 & Sy1 \\
\hline NGC 2273 & $06: 50: 08.7$ & $+60: 50: 45$ & 13.54 & 0.006 & Sy2 & Mrk 501 & $16: 53: 52.2$ & $+39: 45: 36$ & 13.78 & 0.033 & BL Lac \\
\hline $1 \mathrm{H} 0707-495$ & $07: 08: 41.5$ & $-49: 33: 06$ & 15.70 & 0.041 & NLSy1 & MCG +05-40-026 & $17: 01: 07.8$ & $+29: 24: 25$ & 15.78 & 0.036 & NLSy1 \\
\hline IRAS $07245-3548$ & $07: 26: 26.3$ & $-35: 54: 22$ & 16.80 & 0.029 & Sy2 & NGC 6300 & $17: 16: 59.2$ & $-62: 49: 05$ & 13.08 & 0.003 & Sy 2 \\
\hline Mrk 9 & $07: 36: 57.0$ & $+58: 46: 13$ & 14.37 & 0.039 & Sy1 & PDS 456 & $17: 28: 19.9$ & $-14: 15: 56$ & 14.03 & 0.184 & NLSy1 \\
\hline IRAS $07378-3136$ & $07: 39: 44.7$ & $-31: 43: 02$ & $* 16.8$ & 0.025 & Sy2 & IGR J18244-5622 & $18: 24: 19.4$ & $-56: 22: 09$ & 14.40 & 0.017 & Sy2 \\
\hline Mrk 1210 & 08:04:05.9 & $+05: 06: 50$ & 13.70 & 0.013 & Sy2 & LEDA 3097193 & $18: 26: 32.4$ & $+32: 51: 30$ & $\ldots$. & 0.022 & Sy2 \\
\hline FAIRALL 0272 & $08: 23: 01.1$ & $-04: 56: 05$ & 16.00 & 0.021 & Sy2 & $3 \mathrm{C} 382$ & $18: 35: 03.4$ & $+32: 41: 47$ & 15.39 & 0.058 & Sy 1 \\
\hline FAIRALL 1146 & $08: 38: 30.8$ & $-35: 59: 33$ & 16.10 & 0.031 & Sy1 & H $1834-653$ & $18: 38: 20.5$ & $-65: 25: 39$ & 14.53 & 0.013 & Sy2 \\
\hline SWIFT J0845.0-3531 & $08: 45: 21.4$ & $-35: 30: 24$ & $\ldots$ & 0.137 & Sy1 & 3C 390.3 & $18: 42: 09.0$ & $+79: 46: 17$ & 15.38 & 0.057 & Sy1 \\
\hline MCG +01-24-012 & $09: 20: 46.2$ & $-08: 03: 21$ & 13.70 & 0.020 & Sy2 & 2 E $1849.2-7832$ & $18: 57: 07.7$ & $-78: 28: 21$ & $* 14.5$ & 0.042 & Sy1 \\
\hline MCG +04-22-042 & $09: 23: 43.1$ & $+22: 54: 33$ & 14.80 & 0.033 & NLSy1 & IGR J19473+4452 & $19: 47: 19.4$ & $+44: 49: 42$ & 15.70 & 0.053 & Sy2 \\
\hline MCG $-05-23-16$ & $09: 47: 40.2$ & $-30: 56: 54$ & 13.69 & 0.008 & Sy1 & $3 \mathrm{C} 403$ & $19: 52: 15.9$ & $+02: 30: 24$ & 16.50 & 0.059 & Sy2 \\
\hline $3 \mathrm{C} 227$ & $09: 47: 45.1$ & $+07: 25: 20$ & 16.97 & 0.086 & Sy1 & MCG $+07-41-003$ & $19: 59: 28.3$ & $+40: 44: 02$ & 15.10 & 0.056 & Sy2 \\
\hline NGC 3079 & 10:01:58.5 & $+55: 40: 50$ & 12.18 & 0.004 & Sy2 & IGR J20187+4041 & $20: 18: 38.7$ & $+40: 41: 00$ & $\ldots$ & 0.014 & Sy2 \\
\hline Mrk 728 & 11:01:01.8 & $+11: 02: 50$ & 16.93 & 0.036 & Sy2 & IC 5063 & $20: 52: 02.2$ & $-57: 04: 08$ & 13.60 & 0.011 & Sy2 \\
\hline Mrk 421 & $11: 04: 27.2$ & $+38: 12: 32$ & 12.90 & 0.031 & BL Lac & IGR J21277+5656 & $21: 27: 44.9$ & $+56: 56: 40$ & 18.79 & 0.014 & NLSy1 \\
\hline NGC 3516 & $11: 06: 47.4$ & $+72: 34: 07$ & 12.40 & 0.009 & Sy1 & IRAS F21318-2739 & $21: 34: 45.1$ & $-27: 25: 55$ & $* 16.36$ & 0.067 & Sy1 \\
\hline Mrk 732 & $11: 13: 49.8$ & $+09: 35: 10$ & 14.17 & 0.030 & Sy1 & PKS 2149-306 & $21: 51: 55.4$ & $-30: 27: 54$ & 17.90 & 2.345 & FSRQ \\
\hline NGC 4051 & 12:03:09.6 & $+44: 31: 53$ & 12.92 & 0.002 & NLSy1 & PKS 2155-304 & $21: 58: 52.0$ & $-30: 13: 32$ & 13.09 & 0.116 & BL Lac \\
\hline NGC 4151 & $12: 10: 32.5$ & $+39: 24: 21$ & 11.85 & 0.003 & Sy1 & BL Lac & $22: 02: 43.3$ & $+42: 16: 39$ & 14.72 & 0.069 & BL Lac \\
\hline WAS 49b & $12: 14: 17.8$ & $+29: 31: 43$ & 15.40 & 0.064 & Sy2 & NGC 7582 & $23: 18: 23.5$ & $-42: 22: 14$ & 13.57 & 0.005 & Sy1 \\
\hline NGC 4395 & $12: 25: 48.9$ & $+33: 32: 48$ & 10.27 & 0.001 & Sy2 & & & & & & \\
\hline
\end{tabular}

rate exceeding the measurement noise in the light curves. $F_{\text {var }}$ is defined as

$F_{\text {var }}=\sqrt{\frac{S^{2}-\bar{\sigma}^{2} \text { err }}{\bar{x}^{2}}}$

where $S^{2}$ is the sample variance, $\bar{x}$ is the arithmetic mean of $x_{i}$ and $\bar{\sigma}^{2}$ err represents the mean square error, given by

$S^{2}=\frac{1}{N-1} \sum_{i=1}^{N}\left(x_{i}-\bar{x}\right)^{2}$

${\overline{\sigma^{2}}}_{\mathrm{err}}=\frac{1}{N} \sum_{i=1}^{N} \sigma_{\mathrm{err}, \mathrm{i}}^{2}$

The uncertainty in $F_{\text {var }}$ is given by

$\operatorname{err}\left(F_{\mathrm{var}}\right)=\sqrt{\left(\sqrt{\frac{1}{2 N}} \frac{\sigma^{2} \overline{\mathrm{err}}^{2} F_{\mathrm{var}}}{{ }^{2}}+\left(\sqrt{\frac{\bar{\sigma}^{2} \mathrm{err}}{N}} \frac{1}{\bar{x}}\right)^{2}\right.}$

$F_{\text {var }}$ and $\operatorname{err}\left(F_{\text {var }}\right)$ were calculated for each binned light curve. Variability analysis of the sample was carried out in soft $(3-10 \mathrm{keV})$, hard $(10-79 \mathrm{keV})$ and total $(3-79 \mathrm{keV})$ bands. An object is considered variable if $F_{\text {var }}$ (significant at $1 \sigma$ ) is greater than zero. Calculated values of $F_{\text {var }}$ for sources that are found to be variable are given in Tables 2, $3,4,5$ and 6 for BL Lacs, FSRQs, Seyfert 1 galaxies, Seyfert 2 galaxies and NLSy1 galaxies respectively. About $65 \%$ of the sources in our sample are found to be variable.

The number of BL Lacs, FSRQs and NLSy1 galaxies studied here is small (compared to Seyfert 1 and 2 galaxies) to consider the variability shown by these objects as a representation of their class as a whole. Nevertheless, we calculated the weighted mean variability of the different classes of AGN in different X-ray bands namely soft, hard and total bands and the results are presented in Table 7 . In the total NuSTAR band we find an average $F_{\text {var }}$ of $0.310 \pm 0.138$ and $0.093 \pm 0.047$ for BL Lacs and FSRQs respectively. In the radio-quiet category, the average $F_{\text {var }}$ values for Seyfert 1 and Seyfert 2 galaxies are $0.092 \pm 0.061$ and $0.149 \pm 0.108$ respectively. The histogram and cumulative distribution of $F_{\text {var }}$ in the total band for different classes of AGN are shown in Figure 3.

To check for the robustness of the differences in the weighted mean $F_{\text {var }}$ values of different classes of AGN, we carried out two non-parametric statistical tests, namely the Mann-Whitney U test (hereafter referred to as the U test) 

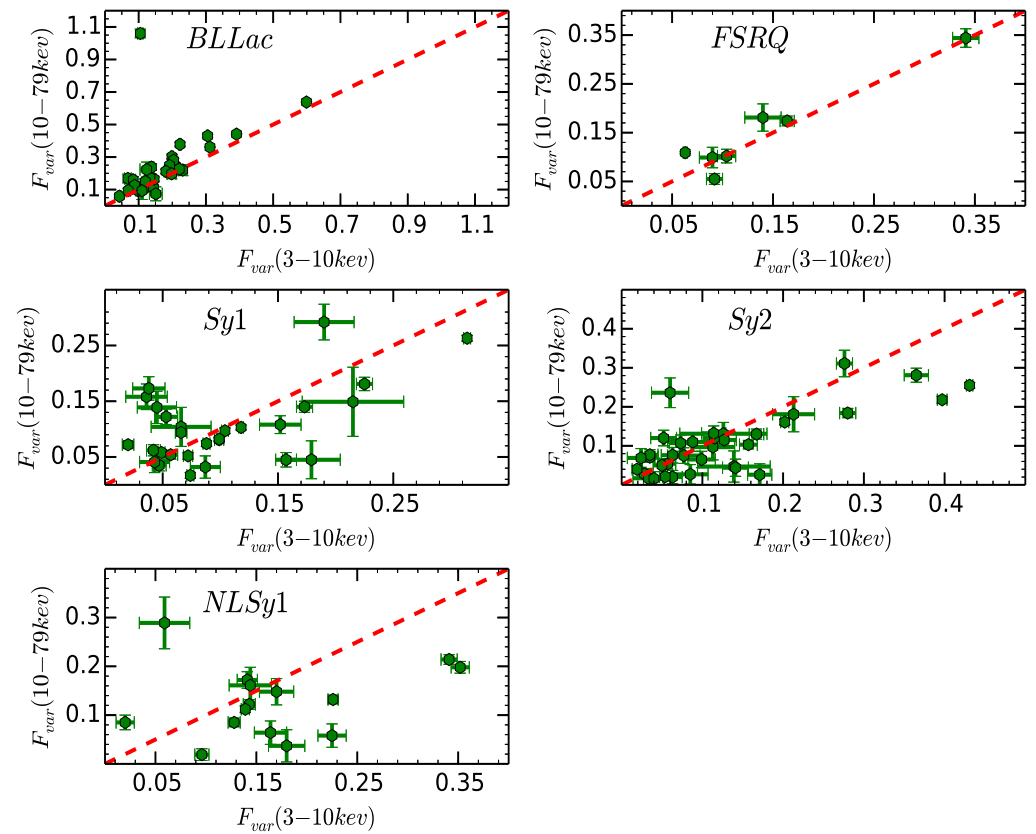

Figure 4. Correlation of flux variations between soft and hard bands for the different classes of AGN. The dashed lines have a slope of

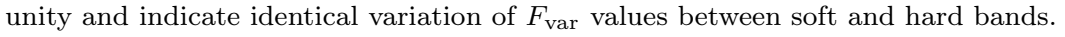

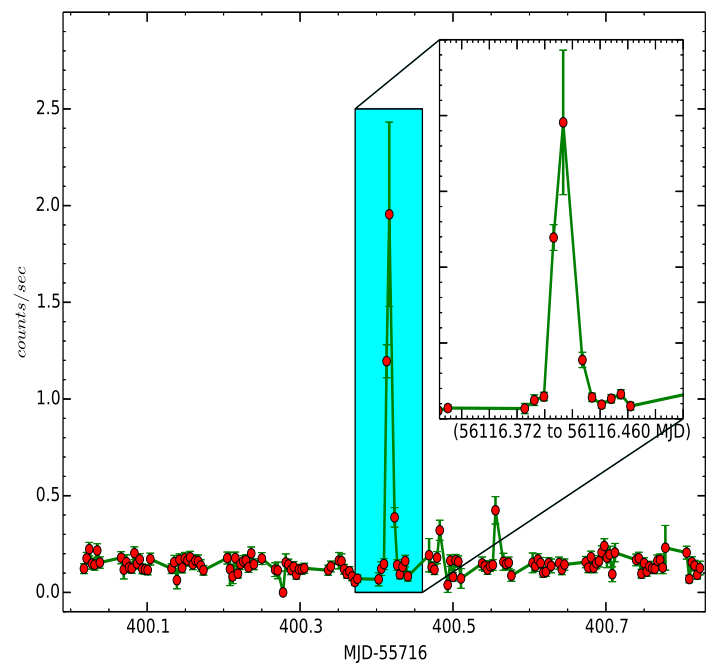

Figure 5. NuSTAR light curve of the BL Lac PKS 2155-304 in the energy range $10-79 \mathrm{keV}$. The inset shows the zoomed in section of the light curve around MJD 56116.372 to 56116.460 where the shortest doubling time of $1.65 \pm 0.16$ is observed.

and the Kolmogorov-Smirnov test (hereafter referred to as the KS test). The U-test is based on the rank of observations rather than the observations themselves. It allows two groups or conditions to be compared without making the assumption that the values are normally distributed. The null hypothesis that is tested in U-test is that the distribu-

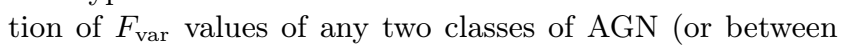
different energy bands in a particular class of AGN) that is compared is identical. The null hypothesis is rejected (at a particular level of confidence) if the U-statistics is less than the critical U-value $\left(U_{\text {crit }}\right)$. The KS test similar to the U-test is also a non-parametric test that can determine if two data sets differ significantly. In this statistics the cumulative distribution of the two data sets that is to be tested are plotted and KS test uses the maximum vertical deviation between the two curves to give the statistics D. The null hypothesis here is that the two data sets that are compared are from the same distribution. This null hypothesis is rejected if $\mathrm{D}$ is greater than the critical $\mathrm{D}$ value $\left(D_{\text {crit }}\right)$. The critical values $U_{\text {crit }}$ and $D_{\text {crit }}$ are evaluated at the $5 \%$ confidence level. In Table 8 we give the results of the two test statistics along with their corresponding $\mathrm{P}$ values for the various comparisons that are studied for the different classes of AGN. From Table 8 it is evident that both $\mathrm{U}$ and KS tests reject the null hypothesis of any differences in the variability properties between FSRQs-BL Lacs and Sy1-Sy2 galaxies in soft, hard and total energy bands. However, comparing Seyfert galaxies (including Seyfert 1 and 2) and blazars (that include FSRQs and BL Lacs), statistical tests clearly indicate that the blazar sources in our sample are more variable than Seyfert galaxies, in all the three energy bands investigated here, namely soft, hard and the full NuSTAR band.

\subsection{Flux variations between soft and hard bands}

To compare the relation between flux variations in the soft $(3-10 \mathrm{keV})$ and hard $(10-79 \mathrm{keV}) \mathrm{X}$-ray bands, $F_{\text {var }}$ was evaluated for the variable sources in the soft and hard bands. In general, for the different types of AGN studied in this work, the derived values of $F_{\text {var }}$ do not show an one to one correspondence between the two energy bands as evident from Figure 4. In the case of BL Lac objects, we have a total of 30 sets of observations on 3 objects. Considering these observations in total, we find that for $90 \%$ of the ob- 
Table 2. Characteristics of flux variations of BL Lac objects

\begin{tabular}{|c|c|c|c|c|c|c|c|}
\hline Name & Type & OBS ID & Obs. date & $\begin{array}{l}\text { Exposure } \\
\text { (secs) }\end{array}$ & $3-10 \mathrm{keV}$ & $\begin{array}{c}\text { var } \pm \operatorname{err}\left(F_{\text {var }}\right) \\
10-79 \mathrm{keV}\end{array}$ & $3-79 \mathrm{keV}$ \\
\hline \multirow[t]{22}{*}{ Mrk 421} & \multirow[t]{22}{*}{ BL Lac } & 10002015001 & 2012-07-07 & 42034 & $0.209 \pm 0.003$ & $0.247 \pm 0.008$ & $0.225 \pm 0.004$ \\
\hline & & 10002016001 & 2012-07-08 & 24885 & $0.305 \pm 0.003$ & $0.430 \pm 0.009$ & $0.358 \pm 0.004$ \\
\hline & & 60002023002 & 2013-01-02 & 9152 & $0.068 \pm 0.010$ & $0.168 \pm 0.027$ & $0.088 \pm 0.013$ \\
\hline & & 60002023004 & 2013-01-10 & 22633 & $0.119 \pm 0.008$ & $0.146 \pm 0.021$ & $0.132 \pm 0.010$ \\
\hline & & 60002023006 & 2013-01-15 & 24182 & $0.199 \pm 0.004$ & $0.302 \pm 0.012$ & $0.243 \pm 0.005$ \\
\hline & & 60002023008 & 2013-01-20 & 24968 & $0.084 \pm 0.007$ & $0.159 \pm 0.020$ & $0.095 \pm 0.009$ \\
\hline & & 60002023010 & 2013-02-06 & 19307 & $0.097 \pm 0.005$ & $0.095 \pm 0.014$ & $0.108 \pm 0.006$ \\
\hline & & 60002023012 & 2013-02-12 & 14780 & $0.204 \pm 0.005$ & $0.280 \pm 0.012$ & $0.241 \pm 0.006$ \\
\hline & & 60002023014 & $2013-02-16$ & 17359 & $0.231 \pm 0.013$ & $0.220 \pm 0.034$ & $0.237 \pm 0.020$ \\
\hline & & 60002023016 & 2013-03-04 & 17252 & $0.122 \pm 0.005$ & $0.115 \pm 0.016$ & $0.122 \pm 0.007$ \\
\hline & & 60002023018 & 2013-03-11 & 17474 & $0.144 \pm 0.005$ & $0.112 \pm 0.014$ & $0.125 \pm 0.006$ \\
\hline & & 60002023020 & 2013-03-17 & 16558 & $0.088 \pm 0.004$ & $0.125 \pm 0.012$ & $0.102 \pm 0.006$ \\
\hline & & 60002023022 & 2013-04-02 & 24772 & $0.223 \pm 0.004$ & $0.378 \pm 0.011$ & $0.295 \pm 0.006$ \\
\hline & & 60002023024 & $2013-04-10$ & 5758 & $0.146 \pm 0.005$ & $0.164 \pm 0.013$ & $0.165 \pm 0.006$ \\
\hline & & 60002023025 & 2013-04-11 & 57509 & $0.599 \pm 0.001$ & $0.638 \pm 0.004$ & $0.621 \pm 0.002$ \\
\hline & & 60002023027 & 2013-04-12 & 7630 & $0.134 \pm 0.002$ & $0.172 \pm 0.005$ & $0.150 \pm 0.002$ \\
\hline & & 60002023029 & 2013-04-13 & 16510 & $0.221 \pm 0.002$ & $0.226 \pm 0.005$ & $0.224 \pm 0.002$ \\
\hline & & 60002023031 & 2013-04-14 & 15606 & $0.312 \pm 0.001$ & $0.361 \pm 0.002$ & $0.335 \pm 0.001$ \\
\hline & & 60002023033 & 2013-04-15 & 17278 & $0.192 \pm 0.006$ & $0.248 \pm 0.005$ & $0.199 \pm 0.008$ \\
\hline & & 60002023035 & 2013-04-16 & 20279 & $0.391 \pm 0.002$ & $0.441 \pm 0.004$ & $0.414 \pm 0.002$ \\
\hline & & 60002023037 & 2013-04-18 & 17795 & $0.181 \pm 0.005$ & $0.212 \pm 0.013$ & $0.199 \pm 0.006$ \\
\hline & & 60002023039 & 2013-04-19 & 15958 & $0.120 \pm 0.005$ & $0.149 \pm 0.013$ & $0.134 \pm 0.006$ \\
\hline \multirow[t]{3}{*}{ Mrk 501} & \multirow[t]{3}{*}{ BL Lac } & 60002024004 & 2013-05-08 & 26141 & $0.138 \pm 0.007$ & $0.238 \pm 0.019$ & $0.163 \pm 0.008$ \\
\hline & & 60002024006 & 2013-07-12 & 10857 & $0.043 \pm 0.004$ & $0.059 \pm 0.008$ & $0.047 \pm 0.005$ \\
\hline & & 60002024008 & 2013-07-13 & 10343 & $0.069 \pm 0.007$ & $0.094 \pm 0.012$ & $0.081 \pm 0.007$ \\
\hline \multirow[t]{5}{*}{ PKS 2155-304 } & \multirow[t]{5}{*}{ BL Lac } & 10002010001 & 2012-07-08 & 33838 & $0.105 \pm 0.008$ & $1.060 \pm 0.027$ & $0.319 \pm 0.011$ \\
\hline & & 60002022004 & 2013-07-16 & 13856 & $0.124 \pm 0.020$ & $0.223 \pm 0.046$ & $0.134 \pm 0.022$ \\
\hline & & 60002022008 & 2013-08-08 & 13496 & $0.110 \pm 0.040$ & $0.093 \pm 0.054$ & $0.156 \pm 0.054$ \\
\hline & & 60002022012 & $2013-08-26$ & 11356 & $0.198 \pm 0.013$ & $0.197 \pm 0.030$ & $0.209 \pm 0.015$ \\
\hline & & 60002022014 & 2013-09-04 & 12282 & $0.151 \pm 0.019$ & $0.074 \pm 0.043$ & $0.079 \pm 0.024$ \\
\hline
\end{tabular}

Table 3. Characteristics of flux variations of FSRQs

\begin{tabular}{llllllll}
\hline Name & Type & OBS ID & Obs.date & $\begin{array}{l}\text { Exposure } \\
(\text { secs })\end{array}$ & $3-10 \mathrm{keV}$ & $\begin{array}{c}F_{\text {var }} \pm \operatorname{err}\left(F_{\text {var }}\right) \\
10-79 \mathrm{keV}\end{array}$ & $3-79 \mathrm{keV}$ \\
\hline 3C 273 & & & & & & & \\
& FSRQ & 00015013001 & $2012-07-02$ & 2573 & $0.341 \pm 0.013$ & $0.344 \pm 0.019$ & $0.341 \pm 0.012$ \\
& & 00015016001 & $2012-07-02$ & 2990 & $0.140 \pm 0.018$ & $0.181 \pm 0.028$ & $0.164 \pm 0.017$ \\
& & 10002020001 & $2012-07-14$ & 244003 & $0.063 \pm 0.002$ & $0.109 \pm 0.002$ & $0.074 \pm 0.002$ \\
3C 279 & & 10012007001 & $2012-07-13$ & 4530 & $0.090 \pm 0.013$ & $0.099 \pm 0.021$ & $0.096 \pm 0.013$ \\
& FSRQ & 60002020002 & $2013-12-16$ & 39594 & $0.104 \pm 0.009$ & $0.102 \pm 0.014$ & $0.098 \pm 0.009$ \\
PKS 2149-306 & FSRQ & 60001099004 & $2014-04-18$ & 44167 & $0.092 \pm 0.008$ & $0.055 \pm 0.011$ & $0.120 \pm 0.007$ \\
\hline
\end{tabular}

servations, the amplitude of variations in the hard band is larger than the variations in the soft band. The weighted mean $F_{\text {var }}$ for BL Lac objects in the hard and soft bands are $0.340 \pm 0.144$ and $0.342 \pm 0.177$ respectively. Statistical analysis by both $\mathrm{U}$ and KS tests provides no evidence to suggest the variability pattern between soft and hard bands in BL Lacs are different. FSRQs too show similar amplitude of variations within the errors between hard and soft bands and have weighted mean $F_{\text {var }}$ of $0.112 \pm 0.028$ and $0.079 \pm$ 0.045 in hard and soft bands respectively. Among the 28 sets of observations on 17 Seyfert 1 galaxies, in about $36 \%$ of ob- servations the amplitude of flux variations in the hard band are larger than those in the soft band. Similarly, in Seyfert 2 galaxies in 13/36 sets of observations, the hard band shows a higher variability amplitude compared to the soft band. However, based on statistical analysis (Table 8) we find no difference in the variability characteristics between soft and hard bands in Seyfert 1 and 2 galaxies. For NLSy1 galaxies, we have 14 sets of observations on 7 objects. Considering all the 7 objects together, both U and KS tests show that there is no significant difference in the $F_{\text {var }}$ values between soft and hard bands. Of these, we find that in 3 sets of ob- 
servations $(\sim 20 \%)$ the variability amplitude is larger in the hard band than in the soft band pertaining to the sources 1H0323+342, MCG +04-22-042 and PDS 456 .

\subsection{Flux variability time scale}

Knowledge of the time scales on which the X-ray flux varies is very important as it can put constraints on the size of the emission region. For sources that have shown flux variations, we scan their light curves in the energy range $3-10 \mathrm{keV}$ and $10-79 \mathrm{keV}$ to find the time scale of flux variations. For this we calculated the flux doubling time/halving time defined as

$$
F(t)=F\left(t_{0}\right) \cdot 2^{\left(t-t_{0}\right) / \tau}
$$

here, $\tau$ is the characteristic flux doubling/halving time scale and $F\left(t_{0}\right)$ and $F(t)$ are values of the fluxes at time $t_{0}$ and $t$ respectively. This time scale is evaluated by imposing the condition that the difference between the fluxes at times $t_{0}$ and $t$ is greater than $3 \sigma$ (Foschini et al. 2011). The best fit values obtained on fitting Equation 5 to the data are given in Table 9. The quoted uncertainties in $\tau$ are the $1 \sigma$ errors. A total of 13 sources are found to have flux doubling/halving time scale less than $10 \mathrm{~min}$. Such behaviour is not restricted to one AGN type and is seen in all types of AGN. Among the Seyfert 2 galaxies NGC 1365 has shown flux doubling/halving time less than $10 \mathrm{~min}$ in all three epochs it has been observed. The shortest flux doubling/halving time scale measured in the energy range of $10-79 \mathrm{keV}$ is $1.65 \pm 0.16 \mathrm{~min}$ for the BL Lac object PKS 2155-304. The 10-79 keV light curve of the source is shown in Figure 5 and the part of the light curve during the epoch where such doubling time is observed is shown in the inset in Figure 5. This is significantly smaller than that previously observed in the X-ray band (Paliya et al. 2015) in another blazar Mrk 421 and is the shortest flux doubling/halving time in the hard X-ray band ever recorded in PKS 2155-304.

\subsection{Time delay between flux variations in hard and soft bands}

To quantify the degree of correlation between flux variations in soft and hard bands we used a model based approach using the code JAVELIN ${ }^{2}$, a Python based implementation of SPEAR (Stochastic Process Estimation for AGN Reverberation; Zu et al. 2011, 2013). JAVELIN has been designed to improve lag measurements between continuum and line variations in AGN by modelling the light curves using a damped random walk process (DRWP; Kelly et al. 2009; MacLeod et al. 2010). It then compares the simulated light curves with the observed ones to find the lag and this has been found to work well (Pancoast et al. 2014). We applied the JAVELIN method to all the sources in our sample. For all the sources the variations between soft and hard bands are consistent with zero lag. Several studies do exist in the literature on time lags between flux variations in different X-ray bands. For blazars in general using data in the $0.3-10 \mathrm{keV}$ band, soft lags (lower energy variations lagging the higher

2 http://www.astronomy.ohio-state.edu/ yingzu/codes.html energy variations), hard lags (higher energy variations lagging the lower energy variation) and zero lags have been observed (Falcone et al. 2004; Zhang et al. 2006; Brinkmann et al. 2003; Ravasio et al. 2004; Abeysekara et al. 2016). This indicates the complex spectral and temporal characteristics of blazars that show different behaviour at different times. Observations of few Seyfert galaxies in the hard Xray band by SWIFT/BAT in 2 days binned light curve did not show any delay between $20-50$ and $50-100 \mathrm{keV}$ bands (Caballero-Garcia et al. 2012).

\subsection{Duty cycle of flux variations}

To characterize the incidence of observability of X-ray variations in different classes of AGN, we have calculated the duty cycle of X-ray flux variations using the definition of Romero et al. (1999). Since only about $65 \%$ of the sources in our sample has shown variations exceeding the measurement noise characterised by $F_{\text {var }}$, duty cycles are estimated not as a fraction of the variable objects within a given class, but as the ratio of the time over which the objects of a given class are found to vary to the total time of observations carried out on each objects in the class. An approach of this kind will take into account the fact that all AGN do not show variations at all times. Also, as the duration of observation is not the same for all the objects, the contribution to the duty cycle has been weighted by the number of times, as well as the duration each source was observed. Duty cycle is defined as

$D C=\frac{\sum_{i=1}^{n} N_{i}\left(1 / \Delta t_{i}\right)}{\sum_{i=1}^{n}\left(1 / \Delta t_{i}\right)} \times 100 \%$

Here, $\Delta t_{i}=\Delta t_{0}(1+z)^{-1}$, is the duration corrected for cosmological redshift of the source observed and $N_{i}$ equals 1 if the object is variable during the period of observations $\Delta t_{i}$ and 0 otherwise. We find NLSy1 galaxies show the highest DC of $87.14 \%$ followed by BL Lacs $(82.44 \%$ ), Seyfert galaxies (Seyfert 1 and 2 galaxies show similar DC of variability of $55.87 \%$ and $56.47 \%$ respectively) and FSRQs (22.79\%). Most of the BL Lacs belong to the HSP category of AGN and FSRQs are in general LSP sources. In the X-ray band, HSPs are known to be more variable in the X-ray band than LSPs, because, in the former the X-ray spectrum falls in the synchrotron region of the SED, while in the latter, it falls in the IC region. Therefore, the difference in the DC of variability between BL Las and FSRQs is due to the differences in the physical processes that contribute to the X-ray emission in the NuSTAR band in these two classes of AGN.

\subsection{Spectral variations}

The lack of one to one correspondence between the amplitude of flux variations in the soft and hard bands in some sources indicate that they show spectral variations. To further characterise spectral variations, we construct diagrams of hardness ratio (HR) plotted as a function of total flux in the $3-79 \mathrm{keV}$ energy range. This is a model independent way to study spectral variations. HR is estimated using the following relation

$H R=\frac{F_{\text {hard }}}{F_{\text {soft }}}$ 

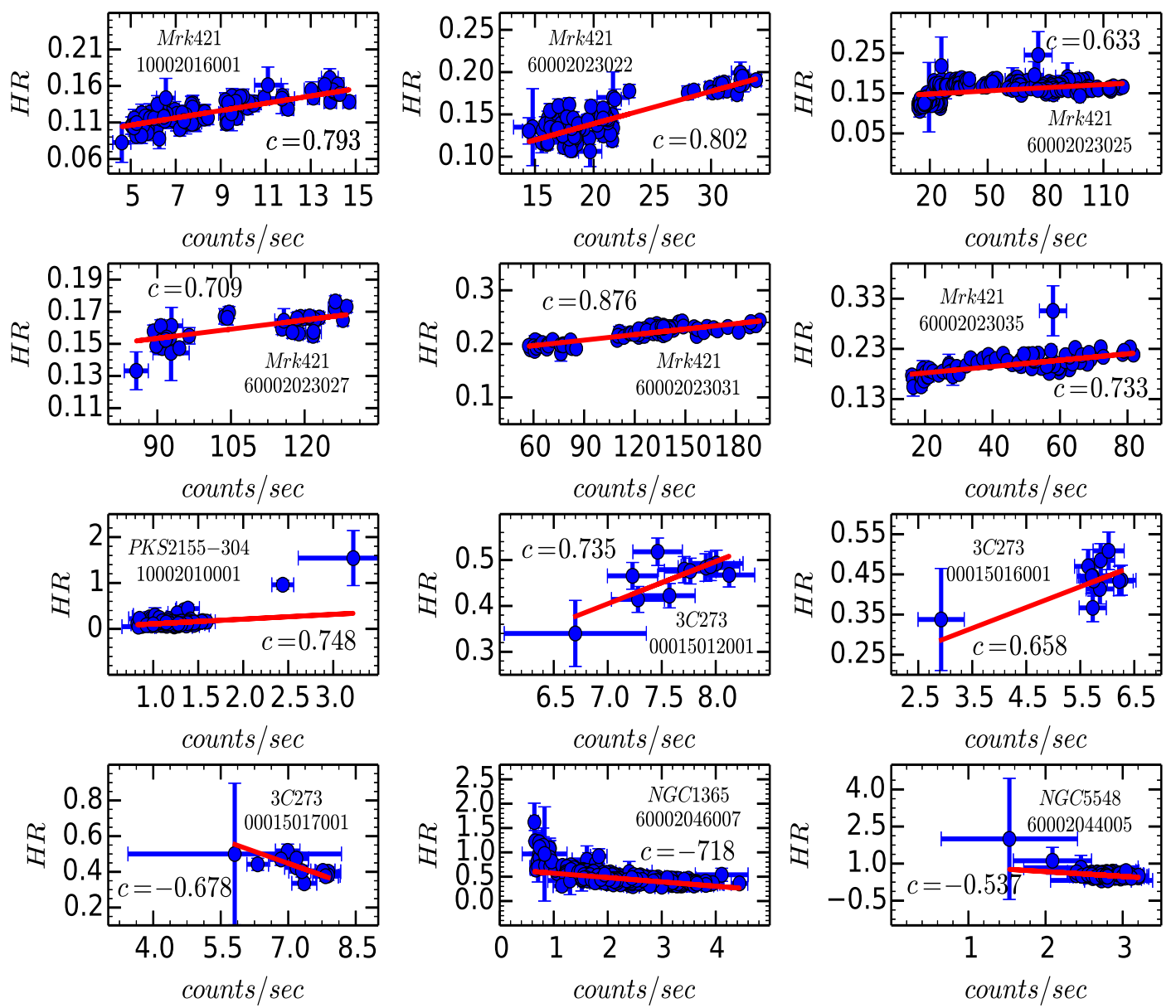

Figure 6. Hardness ratio plotted as a function of count rate in the $3-79 \mathrm{keV}$ energy range. Red solid line is the weighted linear least squares fit to the data. The name of the source, the OBSID and the correlation coefficient are given in each panel.
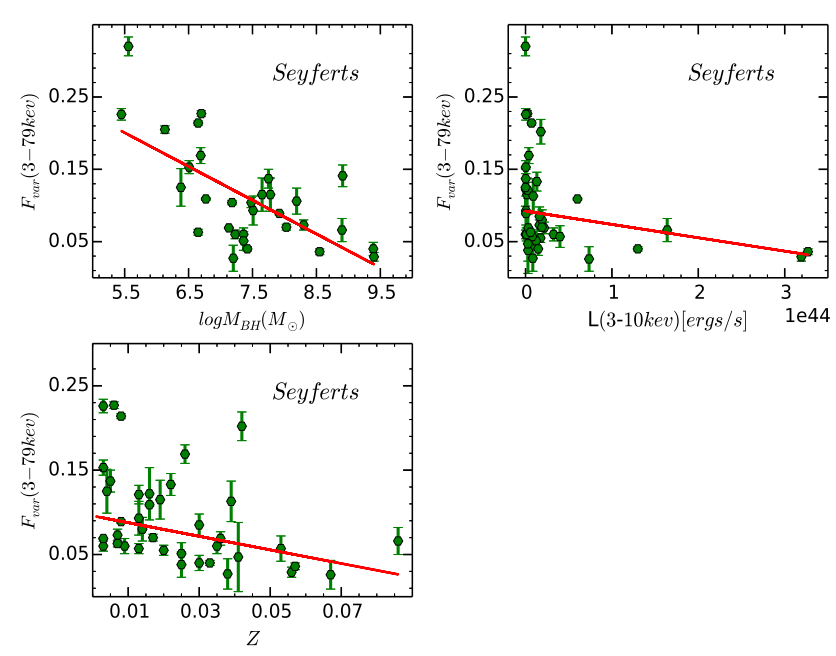

Figure 7. Correlation of $F_{\text {var }}$ with $\mathrm{BH}$ mass, redshift and luminosity in the $3-10 \mathrm{keV}$ band. where, $F_{\text {hard }}$ and $F_{\text {soft }}$ refer to the fluxes in the $10-79 \mathrm{keV}$ and the $3-10 \mathrm{keV}$ respectively. For most of the sources we do not find a significant correlation between variations in HR and total flux. However, for some sources we do find a correlation. For those sources, the plot of HR as a function of the flux in the $3-79 \mathrm{keV}$ band is shown in Figure 6 . To quantify the significance of the correlation, we fit the observed points in the HR $\mathrm{v} / \mathrm{s}$ flux diagram using a linear function of the form $H R=a \times f l u x_{3-79 k e V}+b$. During the fit we take into account the errors both in HR and flux following Press et al. (1992). The results of the fit are given in Table 10 and they are shown as solid lines in Figure 6. Significant spectral variations are seen in the BL Lacs objects Mrk 421 and PKS 2155-304, the FSRQ 3C 273, the Seyfert 1 galaxy NGC 5548 and the Seyfert 2 galaxy NGC 1365. For the BL Lac object Mrk 421 a significant harder when brighter trend is seen in 6 epochs of observations. However, for the FSRQ 3C 273, in the three epochs where a correlation between HR and total flux is found, on two epochs a harder when brighter trend is found, while, in one epoch a softer when brighter trend is noticed. Blazars in general are found to show a harder when brighter behaviour. Such hardening when brightening behaviour more often seen in the HSP category (Giommi et al. 1990; Pian et al. 1998; 
Table 4. Characteristics of flux variations of Seyfert 1 galaxies

\begin{tabular}{|c|c|c|c|c|c|c|c|}
\hline Name & Type & OBS ID & Obs.date & $\begin{array}{l}\text { Exposure } \\
\text { (secs) }\end{array}$ & \multicolumn{3}{|c|}{$F_{\mathrm{var}} \pm \operatorname{err}\left(F_{\mathrm{var}}\right)$} \\
\hline \multirow[t]{3}{*}{ Mrk 335} & \multirow[t]{3}{*}{ Sy1 } & 60001041002 & 2013-06-13 & 21299 & $0.190 \pm 0.026$ & $0.292 \pm 0.032$ & $0.204 \pm 0.022$ \\
\hline & & 60001041003 & 2013-06-13 & 21525 & $0.179 \pm 0.025$ & $0.045 \pm 0.034$ & $0.188 \pm 0.023$ \\
\hline & & 60001041005 & 2013-06-25 & 93028 & $0.157 \pm 0.009$ & $0.045 \pm 0.013$ & $0.114 \pm 0.008$ \\
\hline \multirow[t]{2}{*}{$3 \mathrm{C} 120$} & \multirow[t]{2}{*}{ Sy1 } & 60001042002 & 2013-02-06 & 21606 & $0.047 \pm 0.006$ & $0.035 \pm 0.010$ & $0.036 \pm 0.006$ \\
\hline & & 60001042003 & 2013-02-06 & 127731 & $0.074 \pm 0.003$ & $0.017 \pm 0.005$ & $0.044 \pm 0.003$ \\
\hline Mrk 9 & Sy1 & 60061326002 & 2013-10-29 & 23310 & $0.066 \pm 0.026$ & $0.104 \pm 0.035$ & $13 \pm 0.024$ \\
\hline \multirow{2}{*}{$\mathrm{MCG}-05-23-16$} & \multirow[t]{2}{*}{ Sy1 } & 10002019001 & 2012-07-16 & 33927 & $0.088 \pm 0.003$ & $0.074 \pm 0.005$ & $0.082 \pm 0.003$ \\
\hline & & 60001046002 & 2013-06-03 & 160478 & $0.104 \pm 0.003$ & $0.097 \pm 0.005$ & $0.096 \pm 0.003$ \\
\hline $3 \mathrm{C} 227$ & Sy1 & 60061329004 & 2014-02-26 & 12064 & $0.036 \pm 0.018$ & $0.158 \pm 0.023$ & $0.066 \pm 0.016$ \\
\hline NGC 3516 & Sy1 & 60002042004 & 2014-07-11 & 72089 & $0.053 \pm 0.010$ & $0.122 \pm 0.014$ & $0.060 \pm 0.009$ \\
\hline Mrk 732 & Sy1 & 60061208002 & 2013-06-11 & 26359 & $0.043 \pm 0.013$ & $0.041 \pm 0.019$ & $0.085 \pm 0.013$ \\
\hline \multirow[t]{3}{*}{ NGC 4151} & \multirow[t]{3}{*}{ Sy1 } & 60001111002 & 2012-11-12 & 21864 & $0.049 \pm 0.003$ & $0.058 \pm 0.004$ & $0.050 \pm 0.002$ \\
\hline & & 60001111003 & 2012-11-12 & 57036 & $0.099 \pm 0.004$ & $0.081 \pm 0.003$ & $0.069 \pm 0.003$ \\
\hline & & 60001111005 & 2012-11-14 & 61531 & $0.099 \pm 0.002$ & $0.082 \pm 0.003$ & $0.088 \pm 0.002$ \\
\hline Mrk 231 & Sy1 & 60002025004 & 2013-05-09 & 28557 & $0.215 \pm 0.044$ & $0.149 \pm 0.062$ & \pm 0.041 \\
\hline \multirow[t]{3}{*}{ MCG $-06-30-15$} & \multirow[t]{3}{*}{ Sy1 } & 60001047002 & 2013-01-29 & 23270 & $0.225 \pm 0.007$ & $0.181 \pm 0.012$ & $0.199 \pm 0.007$ \\
\hline & & 60001047003 & 2013-01-30 & 127232 & $0.314 \pm 0.003$ & $0.263 \pm 0.005$ & $0.289 \pm 0.003$ \\
\hline & & 60001047005 & 2013-02-02 & 29646 & $0.173 \pm 0.007$ & $0.140 \pm 0.010$ & $0.154 \pm 0.006$ \\
\hline NGC 5506 & Sy1 & 60061323002 & 2014-04-01 & 56585 & $0.072 \pm 0.004$ & $0.052 \pm 0.006$ & $0.063 \pm 0.004$ \\
\hline \multirow[t]{2}{*}{ NGC 5548} & \multirow[t]{2}{*}{ Sy1 } & 60002044006 & 2013-09-10 & 51460 & $0.066 \pm 0.005$ & $0.094 \pm 0.007$ & $0.066 \pm 0.005$ \\
\hline & & 60002044008 & 2013-12-20 & 50103 & $0.057 \pm 0.006$ & $0.054 \pm 0.008$ & $0.073 \pm 0.006$ \\
\hline \multirow[t]{2}{*}{ Mrk 290} & \multirow[t]{2}{*}{ Sy1 } & 60061266002 & 2013-11-14 & 25012 & $0.087 \pm 0.013$ & $0.032 \pm 0.020$ & $0.023 \pm 0.013$ \\
\hline & & 60061266004 & 2013-11-27 & 26348 & $0.038 \pm 0.014$ & $0.173 \pm 0.021$ & $0.057 \pm 0.013$ \\
\hline \multirow[t]{2}{*}{ 3C 390.3} & \multirow[t]{2}{*}{ Sy1 } & 60001082002 & 2013-05-24 & 23643 & $0.042 \pm 0.006$ & $0.062 \pm 0.010$ & $0.053 \pm 0.006$ \\
\hline & & 60001082003 & 2013-05-24 & 47559 & $0.020 \pm 0.005$ & $0.072 \pm 0.007$ & $0.020 \pm 0.005$ \\
\hline IRAS F21318-2739 & Sy1 & 60061306002 & 2013-10-22 & 19809 & $0.045 \pm 0.017$ & $0.139 \pm 0.029$ & $0.026 \pm 0.017$ \\
\hline NGC 7582 & Sy1 & 60061318002 & 2012-08-31 & 16463 & $0.152 \pm 0.018$ & $0.108 \pm 0.016$ & $0.137 \pm 0.013$ \\
\hline IC $4329 A$ & Sy1 & 60001045002 & 2012-08-12 & 162399 & $0.118 \pm 0.002$ & $0.103 \pm 0.004$ & $0.109 \pm 0.002$ \\
\hline
\end{tabular}
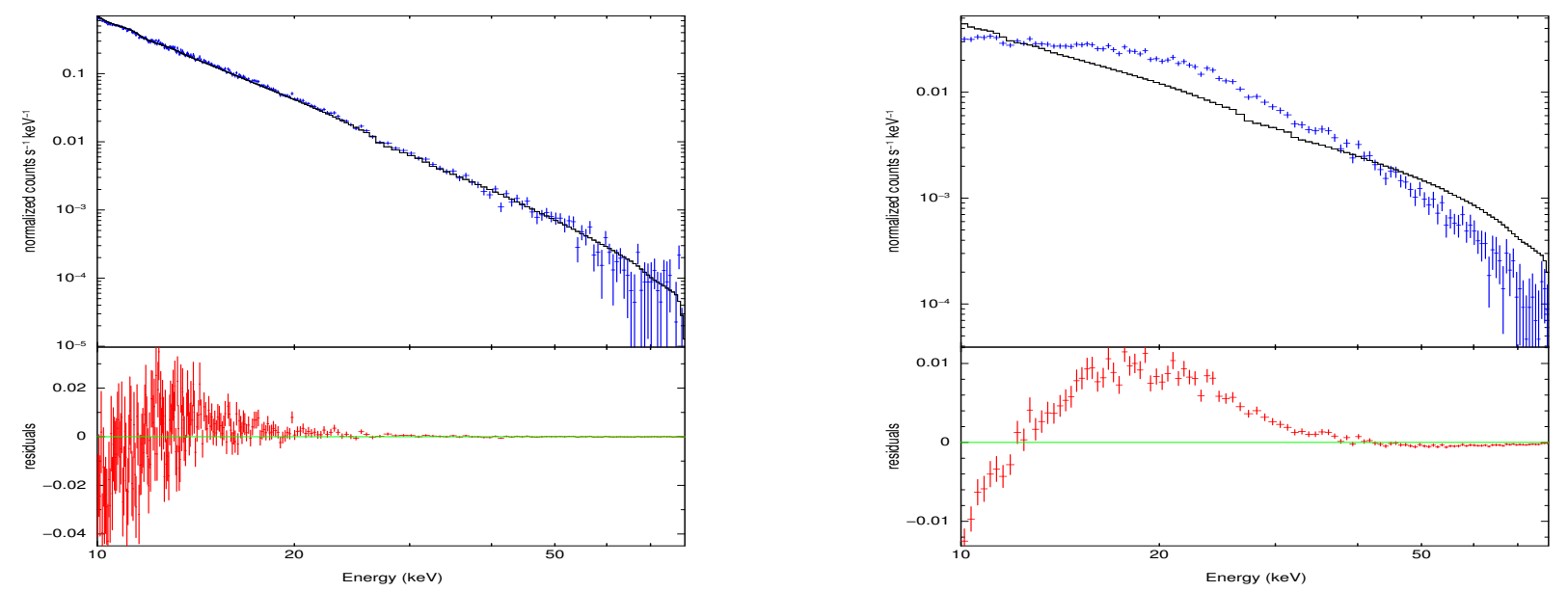

Figure 8. Hard X-ray spectrum of the BL Lac Mrk 421 (left) and the Seyfert 1 galaxy PKS 1409-651 (right) fitted with a power law model along with the residuals.

Giommi et al. 1990; Pian et al. 1998) among other things could be due to the shift of their broad band SEDs to higher energies (Brinkmann et al. 2003). The behaviour seen in Mrk 421 here is observed before as well (Takahashi et al. 1996). For the source PKS 2155-304 too, we find a hardening when brightening trend, also noted earlier in XMM observations (Zhang et al. 2006). In the radio-quiet category, two Seyfert galaxies, namely NGC 5548 and NGC 1365 showed spectral variations with their spectra becoming softer with increasing brightness. This trend is also known in other Seyfert galaxies based on observations from RXTE (Sobolewska \& Papadakis 2009), Swift/XRT (Connolly et al. 2016) and Swift/BAT (Caballero-Garcia et al. 2012), which is expected in various models (Haardt et al. 1997; Turner \& Miller 2009). We con- 
Table 5. Characteristics of flux variations of Seyfert 2 galaxies

\begin{tabular}{|c|c|c|c|c|c|c|c|}
\hline Name & Type & OBS ID & Obs.date & $\begin{array}{l}\text { Exposure } \\
\text { (secs) }\end{array}$ & \multicolumn{3}{|c|}{$F_{\mathrm{var}} \pm \operatorname{err}\left(F_{\mathrm{var}}\right)$} \\
\hline NGC 513 & Sy2 & 60061012002 & 2013-02-16 & 16040 & $0.113 \pm 0.027$ & $0.097 \pm 0.033$ & $0.115 \pm 0.023$ \\
\hline NGC 788 & Sy2 & 60061018002 & 2013-01-28 & 15411 & $0.088 \pm 0.031$ & $0.110 \pm 0.024$ & $0.093 \pm 0.020$ \\
\hline \multirow[t]{3}{*}{ NGC 1068} & Sy2 & 60002030002 & $2012-12-18$ & 57851 & $0.057 \pm 0.010$ & $0.054 \pm 0.013$ & $0.027 \pm 0.008$ \\
\hline & & 60002030004 & $2012-12-20$ & 48560 & $0.035 \pm 0.010$ & $0.071 \pm 0.013$ & $0.065 \pm 0.009$ \\
\hline & & 60002030006 & $2012-12-21$ & 19461 & $0.052 \pm 0.016$ & $0.120 \pm 0.020$ & $0.087 \pm 0.013$ \\
\hline \multirow[t]{5}{*}{ NGC 1365} & Sy2 & 60002046002 & $2012-07-25$ & 36258 & $0.276 \pm 0.010$ & $0.311 \pm 0.034$ & $0.215 \pm 0.019$ \\
\hline & & 60002046003 & $2012-07-26$ & 40588 & $0.157 \pm 0.011$ & $0.103 \pm 0.011$ & $0.130 \pm 0.008$ \\
\hline & & 60002046005 & $2012-12-24$ & 66297 & $0.202 \pm 0.005$ & $0.161 \pm 0.007$ & $0.172 \pm 0.004$ \\
\hline & & 60002046007 & 2013-01-23 & 73650 & $0.431 \pm 0.004$ & $0.255 \pm 0.007$ & $0.338 \pm 0.004$ \\
\hline & & 60002046009 & 2013-02-12 & 69877 & $0.397 \pm 0.006$ & $0.218 \pm 0.009$ & $0.280 \pm 0.006$ \\
\hline $\mathrm{MCG}+03-13-01$ & Sy2 & 60061051002 & 2014-03-18 & 20088 & $0.139 \pm 0.045$ & $0.047 \pm 0.040$ & $0.122 \pm 0.031$ \\
\hline XSS J05054-2348 & Sy2 & 60061056002 & 2013-08-21 & 21161 & $0.035 \pm 0.010$ & $0.077 \pm 0.013$ & $0.060 \pm 0.009$ \\
\hline \multirow[t]{2}{*}{ IRAS $05189-2524$} & Sy2 & 60002027002 & 2013-02-20 & 23141 & $0.060 \pm 0.023$ & $0.236 \pm 0.038$ & $0.177 \pm 0.023$ \\
\hline & & 60002027004 & 2013-10-02 & 25370 & $0.213 \pm 0.026$ & $0.181 \pm 0.045$ & $0.227 \pm 0.025$ \\
\hline NGC 2110 & Sy2 & 60061061004 & 2013-02-14 & 12019 & $0.028 \pm 0.008$ & $0.028 \pm 0.011$ & $0.073 \pm 0.007$ \\
\hline IRAS $07378-3136$ & Sy2 & 60061351002 & 2014-04-20 & 23952 & $0.077 \pm 0.020$ & $0.075 \pm 0.020$ & $0.038 \pm 0.015$ \\
\hline Mrk 1210 & Sy2 & 60061078002 & 2012-10-05 & 15447 & $0.115 \pm 0.014$ & $0.120 \pm 0.015$ & $0.121 \pm 0.011$ \\
\hline \multirow[t]{5}{*}{$\mathrm{MCG}+01-24-012$} & Sy2 & 60061091002 & 2013-04-03 & 12376 & $0.063 \pm 0.014$ & $0.076 \pm 0.020$ & $0.048 \pm 0.013$ \\
\hline & & 60061091004 & 2013-04-10 & 9386 & $0.141 \pm 0.016$ & $0.044 \pm 0.022$ & $0.111 \pm 0.014$ \\
\hline & & 60061091006 & 2013-04-18 & 12178 & $0.171 \pm 0.015$ & $0.026 \pm 0.028$ & $0.053 \pm 0.016$ \\
\hline & & 60061091010 & 2013-05-12 & 15334 & $0.050 \pm 0.011$ & $0.050 \pm 0.016$ & $0.024 \pm 0.010$ \\
\hline & & 60061091012 & 2013-05-22 & 12289 & $0.063 \pm 0.012$ & $0.020 \pm 0.017$ & $0.038 \pm 0.011$ \\
\hline NGC 3079 & Sy2 & 60061097002 & 2013-11-12 & 21542 & $0.126 \pm 0.054$ & $0.131 \pm 0.029$ & $0.125 \pm 0.026$ \\
\hline NGC 4395 & Sy2 & 60061322002 & 2013-05-10 & 19249 & $0.365 \pm 0.015$ & $0.281 \pm 0.018$ & $0.320 \pm 0.013$ \\
\hline \multirow[t]{3}{*}{ Mrk 248} & Sy2 & 60061241002 & 2013-04-21 & 12901 & $0.033 \pm 0.019$ & $0.017 \pm 0.025$ & $0.036 \pm 0.017$ \\
\hline & & 60061241004 & 2013-11-17 & 28909 & $0.040 \pm 0.013$ & $0.016 \pm 0.018$ & $0.065 \pm 0.011$ \\
\hline & & 60061241006 & 2013-11-23 & 23056 & $0.127 \pm 0.015$ & $0.115 \pm 0.022$ & $0.107 \pm 0.014$ \\
\hline NGC 5273 & Sy2 & 60061350002 & 2014-07-14 & 21119 & $0.167 \pm 0.009$ & $0.130 \pm 0.013$ & $0.153 \pm 0.009$ \\
\hline NGC 5674 & Sy2 & 60061337002 & 2014-07-10 & 20671 & $0.073 \pm 0.014$ & $0.107 \pm 0.021$ & $0.051 \pm 0.013$ \\
\hline Mrk 477 & Sy2 & 60061255002 & 2014-05-15 & 18076 & $0.085 \pm 0.022$ & $0.027 \pm 0.025$ & $0.027 \pm 0.018$ \\
\hline NGC 6300 & Sy2 & 60061277002 & $2013-02-25$ & 17706 & $0.280 \pm 0.010$ & $0.184 \pm 0.012$ & $0.226 \pm 0.008$ \\
\hline LEDA 3097193 & Sy2 & 60061354002 & 2014-05-19 & 15645 & $0.114 \pm 0.015$ & $0.132 \pm 0.019$ & $0.133 \pm 0.013$ \\
\hline H 1834-653 & Sy2 & 60061288002 & 2013-02-24 & 27391 & $0.054 \pm 0.007$ & $0.021 \pm 0.009$ & $0.057 \pm 0.006$ \\
\hline IGR J19473+4452 & Sy2 & 60061292002 & 2012-11-06 & 18214 & $0.024 \pm 0.017$ & $0.069 \pm 0.024$ & $0.057 \pm 0.015$ \\
\hline $\mathrm{MCG}+07-41-003$ & Sy2 & 60001083004 & 2013-03-01 & 20715 & $0.019 \pm 0.006$ & $0.040 \pm 0.009$ & $0.029 \pm 0.006$ \\
\hline IGR J20187+4041 & Sy2 & 60061297002 & 2013-12-21 & 20967 & $0.099 \pm 0.017$ & $0.065 \pm 0.021$ & $0.080 \pm 0.014$ \\
\hline
\end{tabular}

Table 6. Characteristics of flux variations of NLSy1 galaxies

\begin{tabular}{|c|c|c|c|c|c|c|c|}
\hline Name & Type & OBS ID & Obs.date & $\begin{array}{l}\text { Exposure } \\
\text { (secs) }\end{array}$ & \multicolumn{3}{|c|}{$F_{\text {var }} \pm \operatorname{err}\left(F_{\mathrm{var}}\right)$} \\
\hline $1 \mathrm{H} 0323+342$ & NLSy1 & 60061360002 & 2014-03-15 & 101633 & $0.141 \pm 0.010$ & $0.172 \pm 0.017$ & $0.138 \pm 0.010$ \\
\hline MCG +04-22-042 & NLSy1 & 60061092002 & $2012-12-26$ & 18845 & $0.020 \pm 0.009$ & $0.085 \pm 0.015$ & $0.081 \pm 0.009$ \\
\hline \multirow[t]{5}{*}{ NGC 4051} & NLSy1 & 60001050002 & $2013-06-17$ & 9434 & $0.225 \pm 0.014$ & $0.058 \pm 0.024$ & $0.168 \pm 0.014$ \\
\hline & & 60001050003 & $2013-06-17$ & 45737 & $0.341 \pm 0.006$ & $0.214 \pm 0.010$ & $0.277 \pm 0.006$ \\
\hline & & 60001050005 & 2013-10-09 & 10202 & $0.164 \pm 0.016$ & $0.064 \pm 0.024$ & $0.128 \pm 0.015$ \\
\hline & & 60001050006 & 2013-10-09 & 49621 & $0.352 \pm 0.009$ & $0.198 \pm 0.012$ & $0.273 \pm 0.008$ \\
\hline & & 60001050008 & 2014-02-16 & 56683 & $0.226 \pm 0.005$ & $0.132 \pm 0.009$ & $0.180 \pm 0.005$ \\
\hline IGR J14552-5133 & NLSy1 & 60061259002 & 2013-09-19 & 21943 & $0.170 \pm 0.017$ & $0.148 \pm 0.027$ & $0.160 \pm 0.017$ \\
\hline MCG $+05-40-026$ & NLSy1 & 60061276002 & 2013-12-19 & 20999 & $0.147 \pm 0.019$ & $0.111 \pm 0.029$ & $0.073 \pm 0.018$ \\
\hline PDS 456 & NLSy1 & 60002032010 & $2014-02-26$ & 109717 & $0.144 \pm 0.020$ & $0.161 \pm 0.037$ & $0.180 \pm 0.022$ \\
\hline \multirow{4}{*}{ IGR J21277+5656 } & NLSy1 & 60001110002 & 2012-11-04 & 49202 & $0.143 \pm 0.006$ & $0.122 \pm 0.010$ & $0.138 \pm 0.006$ \\
\hline & & 60001110003 & 2012-11-05 & 28765 & $0.096 \pm 0.007$ & $0.019 \pm 0.012$ & $0.054 \pm 0.007$ \\
\hline & & 60001110005 & 2012-11-06 & 74583 & $0.139 \pm 0.004$ & $0.112 \pm 0.007$ & $0.124 \pm 0.004$ \\
\hline & & 60001110007 & 2012-11-08 & 42110 & $0.128 \pm 0.006$ & $0.085 \pm 0.009$ & $0.101 \pm 0.006$ \\
\hline
\end{tabular}


Table 7. Weighted mean variability characteristics of different classes of AGN. N1 and N2 represent the number of objects and the number of observations respectively.

\begin{tabular}{llllll}
\hline Type & \multirow{2}{*}{ N1 } & \multirow{2}{*}{ 2 } & \multicolumn{3}{c}{$\operatorname{avg}\left(F_{\text {var }} \pm \operatorname{err}\left(F_{\text {var }}\right)\right)$} \\
& & & $3-10 \mathrm{keV}$ & $10-79 \mathrm{keV}$ & $3-79 \mathrm{keV}$ \\
\hline BL_Lac & 3 & 30 & $0.342 \pm 0.177$ & $0.340 \pm 0.144$ & $0.310 \pm 0.138$ \\
FSRQ & 3 & 7 & $0.079 \pm 0.045$ & $0.112 \pm 0.028$ & $0.093 \pm 0.047$ \\
Sy1 & 17 & 28 & $0.108 \pm 0.068$ & $0.089 \pm 0.052$ & $0.092 \pm 0.061$ \\
Sy2 & 23 & 36 & $0.191 \pm 0.156$ & $0.120 \pm 0.082$ & $0.149 \pm 0.108$ \\
NLSy1 & 7 & 14 & $0.177 \pm 0.085$ & $0.122 \pm 0.052$ & $0.151 \pm 0.065$ \\
LSP & 3 & 7 & $0.079 \pm 0.045$ & $0.112 \pm 0.028$ & $0.093 \pm 0.047$ \\
HSP & 3 & 30 & $0.342 \pm 0.177$ & $0.340 \pm 0.144$ & $0.310 \pm 0.138$ \\
Blazars & 6 & 37 & $0.321 \pm 0.185$ & $0.269 \pm 0.160$ & $0.286 \pm 0.148$ \\
Seyferts & 40 & 64 & $0.124 \pm 0.098$ & $0.095 \pm 0.061$ & $0.104 \pm 0.076$ \\
\hline
\end{tabular}

clude that among the sample of sources studied here, only some sources do show spectral variations. The hard X-ray band in Mrk 421 and PKS 2155-304 is primarily dominated by the non-thermal continuum (not necessarily true for the Seyfert galaxies NGC 5548 and NGC 1365), while in the soft X-ray band there can be contribution from different processes such as power law continuum, soft excess, neutral absorption and absorption features from warm absorbers. Thus, the harder when brighter trend seen in Mrk 421 and PKS 2155-304 is most likely due to changes in the power law component in the relativistic jets of these sources.

\section{DISCUSSION}

X-ray flux variations in AGN can be described by a wide variety of physical processes. In the radio-quiet category of objects hard X-rays can be produced by the Comptonization of soft disk photons in the hot plasma above the accretion disk (Haardt \& Maraschi 1993), whereas, in the radio-loud category of AGN, hard X-rays are dominated by inverse Compton scattering of synchrotron photons by relativistic electrons in the jet via Synchrotron Self Compton process and synchrotron radiation. Given the various methods of the production of hard X-rays in AGN, there can be different causes for X-ray flux variations. In this section we discuss the results of our variability analysis, the potential causes of such variations, the nature of X-ray variations as well as their relation to various physical properties of the sources.

\subsection{Flux variability}

From the results of our variability analysis on a large sample of AGN belonging to different categories, we find that a major fraction of $65 \%$ of the sources in our sample show flux variability. Radio-loud objects (blazars) show large amplitude flux variations relative to their radio-quiet counterparts (Seyfert galaxies) in all the three bands, namely soft, hard and total bands. Such large amplitude flux variations are expected if the X-ray emission in radio-loud sources are dominated by radiative processes in their relativistic jets.

A total of 8 NLSy 1 galaxies are in our sample. They are a separate class of AGN with soft X-ray excess (Boller et al. 1996) showing fast large amplitude X-ray flux variations (Pounds et al. 1995; Leighly 1999) and having FWHM of the
$\mathrm{H} \beta$ line less than $2000 \mathrm{~km} \mathrm{~s}^{-1}$ (Osterbrock \& Pogge 1985). Some of the peculiar properties of these sources have been attributed to them having higher accretions rates compared to the conventional broad line Seyfert galaxies (Peterson et al. 2000). Considering all the NLSy1 galaxies studied here as a whole, from statistical tests, no difference is found in the variability behaviour between soft and hard bands. However, three sources, namely $1 \mathrm{H} 0323+342, \mathrm{MCG}+04-22-042$ and PDS 456 show high $F_{\text {var }}$ values in the hard band relative to the soft band. MCG+04-22-042, $1 \mathrm{H} 0323+342$ and PDS 456 are detected at $1.4 \mathrm{GHz}$ with flux densities of $10 \mathrm{mJy}$, $614 \mathrm{mJy}$ and $22 \mathrm{mJy}$ respectively (Condon et al. 1998). As the above three NLSy1 galaxies are detected in the radio band and as they also show more amplitude of variability in the hard band relative to the soft X-ray band, it is likely that these three sources have a significant hard X-ray contribution from relativistic jets. Of them, $1 \mathrm{H} 0323+342$ is a gamma-ray emitting NLSy 1 galaxy (Abdo et al. 2009), suggesting the presence of a relativistic jet in it similar to blazars. Removing these three sources, from the list of NLSy1 galaxies and classifying the remaining sources as radio-quiet NLSy1 galaxies, we find from $U$ test, that in radio-quiet NLSy1 galaxies variations in soft band is more than the variations in hard band. However, KS test report of no difference in $\mathrm{F}_{\text {var }}$ values between hard and soft bands. In the soft X-ray band our statistical tests indicate that radio-quiet NLSy1 galaxies are more variable than Seyfert 1 galaxies with broad optical emission lines similar to that reported by Leighly (1999). However, from both U and KS test it is evident that there is no difference in the $F_{\text {var }}$ statistics in the hard X-ray band between radio-quiet NLSy1 galaxies and their broad line counterparts. This increased $\mathrm{F}_{\text {var }}$ in the soft band in radio-quiet NLSy1 galaxies might be due to them having lower BH masses than broad line Seyfert galaxies in accordance to the negative correlation between $\mathrm{F}_{\mathrm{var}}$ and BH mass (see section 4.3). However, recently from spectro-polarimetric observations of one NLSy1 galaxy PKS 2004-447, Baldi et al. (2016) suggested that the now existing notion of NLSy1 galaxies having low BH masses need not be true and NLSy1 galaxies too have BH masses similar to blazars. If it is indeed the case, then the larger $\mathrm{F}_{\mathrm{var}}$ seen in NLSy1 galaxies compared to their broad line counterparts might be because of them having X-ray flux variations caused by physical processes other than Seyfert 1 galaxies. In radio-quiet objects, no statistically significant differences in flux variations between soft and hard bands is found both in Seyfert 1 and Seyfert 2 galaxies. Also, we find both Seyfert 1 and Seyfert 2 galaxies showing similar $F_{\text {var }}$ characteristics in all the three bands, namely, soft, hard and the full energy bands. Though these results pertain to variations on short time scales, from long term variability studies in the hard X-ray band, Seyfert 2 galaxies are found to be marginally more variable compared to Seyfert 1 galaxies (Beckmann et al. 2007; Soldi et al. 2014).

\subsection{Spectral variability}

To have an idea of the spectral variations of the sources relative to their brightness, correlation between HR and total flux has been studied. The HR is computed from light curves that cover a wide energy range. Therefore the disadvantage in using HR to characterize spectral variations is that they 
Table 8. Results of statistical tests to compare the $F_{\text {var }}$ properties of different classes of AGN

\begin{tabular}{|c|c|c|c|c|c|c|c|c|}
\hline \multirow[t]{2}{*}{ Parameters } & \multicolumn{4}{|c|}{ Mann-Whitney U test } & \multicolumn{4}{|c|}{ Kolmogorov-Smirnov test } \\
\hline & $U_{\text {obs }}$ & $U_{\text {crit }}$ & Null hypothesis & $\mathrm{P}$ & $\mathrm{D}$ & $D_{\text {crit }}$ & Null hypothesis & $\mathrm{P}$ \\
\hline Sy1 \& Sy2 (soft band) & 471.5 & 358.7 & No & 0.66 & 0.1508 & 0.3427 & No & 0.836 \\
\hline Sy1 \& Sy2 (hard band) & 492.5 & 358.7 & No & 0.88 & 0.1587 & 0.3427 & No & 0.787 \\
\hline Sy1 \& Sy2 (full band) & 446.0 & 358.7 & No & 0.43 & 0.2024 & 0.3427 & No & 0.491 \\
\hline BL \& FSRQ (soft band) & 81 & 54 & No & 0.36 & & & & \\
\hline BL \& FSRQ (hard band) & 67 & 54 & No & 0.15 & & & & \\
\hline BL \& FSRQ (full band) & 76 & 54 & No & 0.27 & & & & \\
\hline Sy \& blazars (soft band) & 711 & 905 & Yes & 0.0009 & 0.3691 & 0.280872 & Yes & 0.002 \\
\hline Sy \& blazars (hard band) & 508 & 905 & Yes & 0.0001 & 0.4569 & 0.280872 & Yes & 0.000 \\
\hline Sy \& blazars (full band) & 536.5 & 905 & Yes & 0.0001 & 0.4573 & 0.280872 & Yes & 0.000 \\
\hline NLSy1 (hard v/s soft)* & 59 & 55 & No & 0.0767 & 0.4286 & 0.514032 & No & 0.111 \\
\hline NLSy1 (hard v/s soft) $\dagger$ & 33 & 37.6 & Yes & 0.0264 & 0.5000 & 0.555218 & No & 0.026 \\
\hline FSRQ (hard v/s soft) & 20 & 8 & No & 0.6101 & & & & \\
\hline BL (hard v/s soft) & 335 & 316 & No & 0.0910 & 0.2667 & 0.351151 & No & 0.200 \\
\hline Sy1 (hard v/s soft) & 387 & 271.8 & No & 0.9442 & 0.1071 & 0.363475 & No & 0.995 \\
\hline Sy2 (hard v/s soft) & & 473.5 & & 0.5157 & 0.1111 & 0.320555 & No & 0.971 \\
\hline NLSy1 \& BLSy1 (soft) & 102 & 122.0 & Yes & 0.0128 & 0.5714 & 0.445165 & Yes & 0.002 \\
\hline NLSy1 \& BLSy1 (hard) & 145 & 122.0 & No & 0.177 & 0.3214 & 0.445165 & No & 0.237 \\
\hline NLSy1 \& blazars (soft) & 220 & 165.6 & No & 0.4237 & 0.2896 & 0.4267 & No & 0.306 \\
\hline NLSy1 \& blazars (hard) & 139 & 165.6 & Yes & 0.0117 & 0.4054 & 0.4267 & No & 0.051 \\
\hline
\end{tabular}

${ }^{*}$ Considering all NlSy1 galaxies that include radio-quiet and radio-loud sources. †Considering only radio-quiet NlSy1 galaxies, that include NGC 4051, IGR J14552-5113, MCG +05-40-026 and IGR J21277+5656.

Table 9. The shortest flux doubling/halving time in minutes and its significance.

\begin{tabular}{|c|c|c|c|c|c|c|}
\hline Name & Type & OBSID & $\begin{array}{r}\tau \\
(3-10 \mathrm{keV}) \\
(\mathrm{min} .)\end{array}$ & Sig. & $\begin{array}{r}\tau \\
(10-79 \mathrm{keV}) \\
\text { (min.) }\end{array}$ & Sig. \\
\hline $3 \mathrm{C} 120$ & Sy1 & 60001042002 & $17.36 \pm 5.60$ & 3.11 & $5.25 \pm 2.01$ & 3.33 \\
\hline $\mathrm{MCG}+07-41-003$ & Sy2 & 60001083002 & $16.82 \pm 5.49$ & 3.07 & $9.13 \pm 2.63$ & 3.56 \\
\hline NGC 4051 & NLSy1 & 60001050008 & $6.96 \pm 1.25$ & 5.65 & $5.70 \pm 1.48$ & 3.91 \\
\hline NGC 4151 & Sy1 & 60001111005 & $16.42 \pm 5.78$ & 3.08 & $23.53 \pm 6.36$ & 3.70 \\
\hline \multirow[t]{8}{*}{ Mrk 421} & \multirow[t]{8}{*}{ BLLac } & 10002015001 & $23.40 \pm 6.66$ & 3.52 & $43.40 \pm 13.44$ & 3.85 \\
\hline & & 60002023006 & $22.32 \pm 6.80$ & 3.29 & $3.04 \pm 1.23$ & 3.75 \\
\hline & & 60002023022 & $113.64 \pm 15.26$ & 7.71 & $96.20 \pm 26.51$ & 3.78 \\
\hline & & 60002023025 & $37.08 \pm 8.69$ & 4.27 & $16.59 \pm 4.44$ & 3.98 \\
\hline & & 60002023027 & $55.84 \pm 4.72$ & 11.57 & $47.44 \pm 8.28$ & 5.58 \\
\hline & & 60002023031 & $34.13 \pm 3.47$ & 9.84 & $34.72 \pm 9.89$ & 3.43 \\
\hline & & 60002023033 & $55.96 \pm 16.92$ & 3.31 & $22.33 \pm 6.48$ & 3.45 \\
\hline & & 60002023035 & $25.11 \pm 2.54$ & 9.88 & $25.07 \pm 5.60$ & 4.48 \\
\hline PKS 2155-304 & BLLac & 10002010001 & $10.29 \pm 3.21$ & 3.19 & $1.65 \pm 0.16$ & 11.67 \\
\hline $3 \mathrm{C} 273$ & FSRQ & 10002020001 & $7.29 \pm 3.26$ & 4.49 & $5.13 \pm 0.51$ & 10.14 \\
\hline IC $4329 A$ & Sy1 & 60001045002 & $22.11 \pm 7.27$ & 3.05 & $14.12 \pm 4.19$ & 3.38 \\
\hline MCG $-05-23-16$ & Sy1 & 60001046002 & $99.58 \pm 23.12$ & 4.23 & $3.35 \pm 1.45$ & 3.89 \\
\hline MCG $-06-30-15$ & Sy1 & 60001047003 & $10.45 \pm 3.18$ & 3.30 & $7.89 \pm 2.53$ & 3.15 \\
\hline PDS 456 & NLSy1 & 60002032002 & $4.94 \pm 1.45$ & 3.27 & $1.99 \pm 0.85$ & 3.34 \\
\hline \multirow[t]{2}{*}{ IGR J21277+5656 } & \multirow[t]{2}{*}{ NLSy1 } & 60001110002 & $10.36 \pm 2.84$ & 3.68 & $32.59 \pm 8.63$ & 3.56 \\
\hline & & 60001110005 & $5.78 \pm 1.93$ & 3.79 & $51.47 \pm 17.07$ & 3.04 \\
\hline NGC 1068 & Sy2 & 60002030004 & $17.63 \pm 8.07$ & 3.98 & $5.12 \pm 1.72$ & 3.02 \\
\hline \multirow[t]{3}{*}{ NGC 1365} & \multirow[t]{3}{*}{ Sy2 } & 60002046005 & $10.49 \pm 2.45$ & 4.32 & $5.36 \pm 2.17$ & 3.14 \\
\hline & & 60002046007 & $8.45 \pm 2.16$ & 3.94 & $7.70 \pm 2.83$ & 3.04 \\
\hline & & 60002046009 & $10.56 \pm 3.16$ & 3.35 & $7.76 \pm 2.39$ & 3.28 \\
\hline NGC 1052 & Sy2 & 60061027002 & $3.53 \pm 1.45$ & 3.10 & $1.86 \pm 1.02$ & 3.67 \\
\hline NGC 4395 & Sy2 & 60061322002 & $3.08 \pm 0.44$ & 7.33 & $4.35 \pm 1.08$ & 4.13 \\
\hline
\end{tabular}


Table 10. Results of correlation analysis. Here, $\mathrm{P}$ and $\mathrm{R}$ are the probability of no correlation and the correlation coefficient respectively.

\begin{tabular}{llrrrrr}
\hline Name & OBS ID & Slope & Intercept & $\chi_{\text {red }}^{2}$ & $P$ & $R$ \\
\hline Mrk 421 & 10002016001 & $0.005 \pm 0.000$ & $0.081 \pm 0.003$ & 0.900 & $<10^{-5}$ & 0.793 \\
& 60002023022 & $0.003 \pm 0.000$ & $0.062 \pm 0.006$ & 3.030 & $<10^{-5}$ & 0.802 \\
& 60002023025 & $0.001 \pm 0.000$ & $0.133 \pm 0.000$ & 6.954 & $<10^{-5}$ & 0.524 \\
& 60002023027 & $0.000 \pm 0.000$ & $0.119 \pm 0.000$ & 1.984 & $<10^{-5}$ & 0.709 \\
& 60002023031 & $0.000 \pm 0.000$ & $0.176 \pm 0.000$ & 2.924 & $<10^{-5}$ & 0.876 \\
PKS 2155-304 & 60002023035 & $0.001 \pm 0.000$ & $0.169 \pm 0.000$ & 3.562 & $<10^{-5}$ & 0.646 \\
3C 273 & 10002010001 & $0.103 \pm 0.024$ & $0.006 \pm 0.029$ & 1.995 & $<10^{-5}$ & 0.748 \\
& 00015012001 & $0.085 \pm 0.034$ & $-0.186 \pm 0.262$ & 1.193 & 0.010 & 0.735 \\
NGC 5548 & 00015016001 & $0.049 \pm 0.032$ & $0.168 \pm 0.187$ & 0.984 & 0.029 & 0.658 \\
NGC 1365 & 00015017001 & $-0.085 \pm 0.028$ & $1.038 \pm 0.208$ & 1.809 & 0.015 & -0.678 \\
& 60002044005 & $-0.192 \pm 0.027$ & $1.060 \pm 0.077$ & 1.301 & $<10^{-5}$ & -0.537 \\
& 60002046007 & $-0.093 \pm 0.007$ & $0.679 \pm 0.018$ & 1.890 & $<10^{-5}$ & -0.718 \\
\hline
\end{tabular}

do not identify spectral components that are responsible for the observed variations measured over a band, however, they are the simplest one to study spectral variations in a model independent way. The majority of sources in our sample do not show any correlation between $\mathrm{HR}$ and flux variations. However, some sources do show correlations between flux and spectral variations. In the radio-loud category, objects such as Mrk 421, PKS 2155-304 and 3C 273 (most of the time), showed a spectral hardening with increasing brightness level, i.e, a hardening when brightening trend is noticed. Among the three radio-loud sources that showed a harder when brighter trend, two sources Mrk 421 and PKS 2155-304 belong to the HSP type, where the harder when brighter trend is often seen (Giommi et al. 1990; Pian et al. 1998; Baloković et al. 2016). In the FSRQ 3C 273 (most of the FSRQs are LSP blazars), we find both harder when brighter and softer when brighter trend. We do not as yet have an unambiguous knowledge of the physical parameters that cause spectral variations. However, the harder when brighter trend seen in the three sources studied here, namely, Mrk 421, PKS 2155-304 and 3C 273 might be due to the emergence of a hard X-ray tail produced in the relativistic jets in the high brightness states in these objects. Though the overall X-ray spectrum of HSP blazars is dominated by the high energy tail of synchrotron emission (contrary to LSP blazars which is dominated by IC emission) it has recently been noticed that in the HSP blazar Mrk 421, during in its low brightness state, excess emission is noticed above $20 \mathrm{keV}$ which is attributed to the emergence of the IC emission (Kataoka \& Stawarz 2016). This clearly indicates that phenomena of flux and spectral variability in Mrk 421 and other blazars is complex. To identify the nature of the hard $\mathrm{X}$-ray emission in our sample of radio-loud vis-a-vis radioquiet sources, we generate the NuSTATR hard X-ray spectra and fit them with a simple power law model. The results of this spectral fitting for a BL Lac Mrk 421 and a Seyfert galaxy PKS 1409-651 is shown in Figure 8. From the figure it is clear that the hard X-ray emission in Mrk 421 (and other blazars) is likely dominated either by jet synchrotron or inverse Compton processes or a combination of both assuming a leptonic jet model. For Seyfert galaxies, power law model is not a good fit to the spectra, thus indicating the hard X-ray emission as due to thermal Comptonization processes. The hardening when brightening trend noticed here in the radio-loud objects Mrk 421, PKS 2155-304 and 3C
273 has been reported earlier (Malizia et al. 2000; Gliozzi et al. 2007, 2008). For two Seyfert galaxies, namely NGC 5548 and NGC 3516 a softening when brightening trend is noticed. Such softening when brightening trend is known for other Seyferts at low energies below $1 \mathrm{keV}$ (Sobolewska \& Papadakis 2009) as well as at energies above $2 \mathrm{keV}$ (Soldi et al. 2014).

\section{3 $\quad F_{\text {var }} \mathrm{v} / \mathrm{s}$ black hole mass}

We show in Figure 7 the relation between $F_{\text {var }}$ in the $3-79$ $\mathrm{keV}$ energy range and black hole $(\mathrm{BH})$ mass. The $\mathrm{BH}$ masses are collected from the online $\mathrm{BH}$ mass data base at the Georgia State University ${ }^{3}$, which is a compilation of $\mathrm{BH}$ masses determined from spectroscopic monitoring observations (Bentz \& Katz 2015). Of the 81 objects in our sample, BH masses are available for only 50 objects. Among those 50 objects, only 37 objects are found to be variable and they are thus used to study the correlation if any between $F_{\text {var }}$ and $\mathrm{BH}$ mass. Figure 7 indicates that there is an anti correlation between $F_{\text {var }}$ and BH mass. Both Spearmann rank correlation and Kendalls $\tau$ tests on the data indicate that the anti correlation is significant with $>99.9$ percent. Using a simple linear least squares fit to the data by including the error in $F_{\text {var }}$ we find

$F_{\text {var }}=-(0.047 \pm 0.009) \times \log M_{B H}+(0.457 \pm 0.072)$

Therefore, there is in general a trend for high mass objects to be less variable though there is a large scatter in the $F_{\text {var }}$ $\mathrm{v} / \mathrm{s} \mathrm{M}_{B H}$ diagram. Studies of X-ray variations in the soft $\mathrm{X}$-ray band on a large number of sources do show a negative correlation between $F_{\text {var }}$ and $\mathrm{BH}$ mass similar to the one observed here (Papadakis \& McHardy 1995; O'Neill et al. 2005; Ponti et al. 2012).

\section{$4.4 \quad F_{\text {var }} \mathrm{v} / \mathrm{s} 3-10 \mathrm{keV}$ luminosity}

The relation between $F_{\text {var }}$ and the luminosity of the source in the energy range $3-10 \mathrm{keV}$ is shown in Figure 7 . To determine the flux, we have fitted power law to the observed spectra for all the sources with fixed galactic column density $n_{H}=1.63 \times 10^{20} \mathrm{~cm}^{-2}$. This was then used to calculate the

3 http://www.astro.gsu.edu/AGNmass/ 
luminosity in the $3-10 \mathrm{keV}$ range. From the figure there is a hint that low luminosity objects are more variable than their high luminosity counterparts.

\subsection{Short time scale variability}

Short time scales of flux variations in AGN taken as the flux doubling/halving time scale in this work are more often seen at high energies in the $\gamma$-ray band, for example, 4 min in PKS 2155-304 (Aharonian et al. 2007) and < 15 min in Mrk 421 (Gaidos et al. 1996) and 14 min in Mrk 421 in the NuSTAR band (Paliya et al. 2015). A recent systematic search for flux doubling/halving time scale less than 15 minutes in the soft X-ray $(0.2-10 \mathrm{keV})$ band, from Swift/XRT observation of blazars by Pryal et al. (2015) has yielded a negative result. Observations of short time scale flux variations in the high energy band of the electromagnetic spectrum is very important, as this can set an upper limit on the size of the emitting region $\left(\mathrm{R}_{s}\right)$ via the relation $R_{s}<\delta c \tau_{\text {var }} /(1+z)$ and consequently, can help in constraining the emission processes. Here, $\tau_{v a r}$ is the time scale of variability, $z$ is the redshift of the source and $\delta$ is the Doppler factor. Doppler factor is defined as $\delta^{-1}=\gamma(1-\beta \cos \theta)$, where, $\gamma$ is the Lorentz factor of the jet, $\beta=v / c$ is the ratio of the jet speed to the speed of light in vacuum and $\theta$ is the viewing angle, the angle between the jet and the observers line of sight. In this work, by characterising the variability time scale by either the flux doubling/halving timescale we found the shortest flux doubling/halving time scale of $1.65 \pm 0.16$ min for the BL Lac object PKS 2155-304. Using this observed $\tau_{v a r}$ we find $R_{s}<2.7 \times 10^{13}(\delta / 10) \mathrm{cm}$. Apart from PKS 2155-304, the blazars Mrk 421 and 3C 273 have also shown flux doubling/halving time scale less than $10 \mathrm{~min}-$ utes. Such short time scales of variations are also seen in few radio-quiet sources. In our sample, we were able to derive flux doubling/halving time scale for 16 sources, of which 13 sources have time scales less than $10 \mathrm{~min}$. Thus, this is the first report of the detection of statistically significant hard X-ray flux variability in a large number of AGN with flux doubling/halving time scale less than 10 minutes. The only other report of short time scale flux doubling/halving variability in the hard X-ray band available in the literature is for the source Mrk 421 (Paliya et al. 2015). On analysis of the blazar light curves for which we were able to derive flux doubling time scales, we found that the flares are asymmetric in nature showing a quick rise and slow decay similar to that observed in PKS 2155-304 and shown in Fig. 5. This might be due to the difference between the particle acceleration and synchrotron cooling time scales.

\section{SUMMARY}

In this work we have examined 176 observations of AGN from NUSTAR to search for hard X-ray flux variations in them, characterize their variability and to see how the flux variations are related to other AGN properties. Key findings of this work are summarized below

(i) A total of 81 sources (3 FSRQs, 4 BL Lac objects, 24 Seyfert 1 galaxies, 42 Seyfert 2 galaxies and 8 NLSy1 galaxies) over 176 sets of observations are studied for hard
X-ray flux variability on hour time scales. We find evidence of X-ray flux variations in about $65 \%$ of the sources in our sample.

(ii) NLSy1 galaxies are found to show the highest DC of variability of about $87 \%$ followed by BL Lac objects that show a DC of $82 \%$. Both Seyfert 1 and 2 galaxies show similar DC of about $56 \%$. The lowest DC of variations of about $23 \%$ is shown by FSRQs.

(iii) In the $3-79 \mathrm{keV}$ band, BL Lacs have a weighted mean $F_{\text {var }}$ of $0.310 \pm 0.138$, while FSRQs have a weighted mean $F_{\text {var }}$ of $0.093 \pm 0.047$. In the radio-quiet category, Seyfert 2 galaxies have a higher weighted mean $F_{\text {var }}$ of 0.149 \pm 0.108 relative to Seyfert 1 galaxies that have a weighted mean $F_{\text {var }}$ of $0.092 \pm 0.061$. Both U and KS test reject the null hypothesis of differences in variability between FSRQs and BL Lac as well as Sy1 and Sy2 galaxies. In the soft $\mathrm{X}$-ray band, radio-quiet NLSy1 galaxies are more variable than their broad line counterparts. Although, the BL Lacs and FSRQs examined here are more variable than the NLSy1 galaxies in the hard band, statistically in the soft bands their variations are indistinguishable.

(iv) Majority of the sources do not show any correlation between $\mathrm{HR}$ and count rate in the $3-79 \mathrm{keV}$ energy range suggesting no spectral variability. However, a small fraction of sources show significant correlation between HR and count rate in the $3-79 \mathrm{keV}$ energy range. The BL Lac objects Mrk 421 and PKS 2155-304 showed a hardening when brightening trend and the FSRQ 3C 273 showed both hardening and softening when brightening behaviour. Among the three epochs of observations, for which 3C 273 has shown variability, in two epochs a hardening when brightening trend is observed, while in one epoch a softening when brightening trend is noticed. In the radio-quiet category, two Seyfert galaxies namely, NGC 5548 and NGC 3516 showed a softening when brightening behaviour. This is opposite to that shown by the BL Lac sources Mrk 421 and PKS $2155-304$.

(v) Sources hosted by massive BHs are less variable on hour time scale than their less massive counterparts. Also,

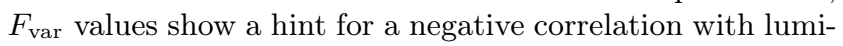
nosity of the sources in the $3-10 \mathrm{keV}$ band.

(vi) We are able to estimate flux doubling/halving time scale for a total of 16 sources in our sample, of which 13 sources show flux doubling/halving time less than 10 minutes. We find the BL Lac PKS 2155-304 to show flux doubling/halving time as short as $1.65 \pm 0.16 \mathrm{~min}$, in the energy range $10-79 \mathrm{keV}$, the shortest ever observed in the X-ray band in any AGN.

We caution on the small number of BL Lacs, FSRQs and NLSy1 galaxies used in this work. Future observations of a large number of BL Lacs, FSRQs and NLSy1 galaxies are indeed needed to confirm the findings reported here.

\section{ACKNOWLEDGMENTS}

We thank the anonymous referee for his/her detailed comments that helped to improve the manuscript. The comments by Dr. Markus Bottcher on an initial version of the manuscript is thankfully acknowledged. This research has made use of data from the NuSTAR mission, a project led by the California Institute of Technology, managed by the Jet 
Propulsion Laboratory and funded by the National Aeronautics and Space Administration. We thank the NuSTAR Operations, Software and Calibration teams for support with the execution and analysis of these observations. This research has made use of the NuSTAR Data Analysis Software (NuSTARDAS) jointly developed by the ASI Science Data Center (ASDC, Italy) and the California Institute of Technology(USA).

\section{REFERENCES}

Abdo A. A., et al., 2009, ApJ, 707, L142

Abdo A. A., et al., 2010, ApJ, 716, 30

Abeysekara A. U., et al., 2016, preprint, (arXiv:1611.04626)

Aharonian F., et al., 2007, ApJ, 664, L71

Aleksić J., et al., 2015, A\&A, 578, A22

Antonucci R., 1993, ARA\&A, 31, 473

Baldi R. D., Capetti A., Robinson A., Laor A., Behar E., 2016, MNRAS, 458, L69

Baloković M., et al., 2016, ApJ, 819, 156

Barthelmy S. D., et al., 2005, Space Sci. Rev., 120, 143

Beckmann V., Shrader C. R., 2012, Active Galactic Nuclei

Beckmann V., Barthelmy S. D., Courvoisier T. J.-L., Gehrels N., Soldi S., Tueller J., Wendt G., 2007, A\&A, 475, 827

Bentz M. C., Katz S., 2015, PASP, 127, 67

Błażejowski M., Sikora M., Moderski R., Madejski G. M., 2000, ApJ, 545, 107

Boettcher M., Mause H., Schlickeiser R., 1997, A\&A, 324, 395

Boller T., Brandt W. N., Fink H., 1996, A\&A, 305, 53

Brinkmann W., Papadakis I. E., den Herder J. W. A., Haberl F., 2003, A\&A, 402, 929

Caballero-Garcia M. D., Papadakis I. E., Nicastro F., Ajello M., 2012, A\&A, 537, A87

Cirasuolo M., Celotti A., Magliocchetti M., Danese L., 2003, MNRAS, 346, 447

Condon J. J., Cotton W. D., Greisen E. W., Yin Q. F., Perley R. A., Taylor G. B., Broderick J. J., 1998, AJ, 115, 1693

Connolly S. D., McHardy I. M., Skipper C. J., Emmanoulopoulos D., 2016, MNRAS, 459, 3963

Dermer C. D., Schlickeiser R., 1993, ApJ, 416, 458

Edelson R., Turner T. J., Pounds K., Vaughan S., Markowitz A., Marshall H., Dobbie P., Warwick R., 2002, ApJ, 568, 610

Falcone A. D., Cui W., Finley J. P., 2004, ApJ, 601, 165

Fiore F., Laor A., Elvis M., Nicastro F., Giallongo E., 1998, ApJ, 503, 607

Foschini L., Ghisellini G., Tavecchio F., Bonnoli G., Stamerra A., 2011, A\&A, 530, A77

Fossati G., et al., 2008, ApJ, 677, 906

Gaidos J. A., et al., 1996, Nature, 383, 319

Gehrels N., et al., 2004, ApJ, 611, 1005

Ghisellini G., Madau P., 1996, MNRAS, 280, 67

Ghisellini G., Haardt F., Matt G., 1994, MNRAS, 267, 743

Giommi P., Barr P., Pollock A. M. T., Garilli B., Maccagni D., 1990, ApJ, 356, 432

Gliozzi M., Brinkmann W., Räth C., Papadakis I. E., Negoro H., Scheingraber H., 2002, A\&A, 391, 875

Gliozzi M., Papadakis I. E., Brinkmann W. P., 2007, ApJ, 656, 691

Gliozzi M., Papadakis I. E., Sambruna R. M., 2008, ApJ, 678, 78

Gupta A. C., Kalita N., Gaur H., Duorah K., 2016, MNRAS, 462, 1508

Haardt F., Maraschi L., 1993, ApJ, 413, 507

Haardt F., Maraschi L., Ghisellini G., 1997, ApJ, 476, 620

Harrison F. A., et al., 2013, ApJ, 770, 103
Heidt J., 1996, in Miller H. R., Webb J. R., Noble J. C., eds, Astronomical Society of the Pacific Conference Series Vol. 110, Blazar Continuum Variability. p. 64

Ivezić Ž., et al., 2002, AJ, 124, 2364

Kataoka J., Stawarz Ł., 2016, ApJ, 827, 55

Kelly B. C., Bechtold J., Siemiginowska A., 2009, ApJ, 698, 895

Konigl A., 1981, ApJ, 243, 700

Leighly K. M., 1999, ApJS, 125, 317

MacLeod C. L., et al., 2010, ApJ, 721, 1014

Malizia A., et al., 2000, MNRAS, 312, 123

Maraschi L., et al., 1999, ApJ, 526, L81

Markowitz A., Edelson R., Vaughan S., 2003, ApJ, 598, 935

McHardy I., 2010, in Belloni T., ed., Lecture Notes in Physics, Berlin Springer Verlag Vol. 794, Lecture Notes in Physics, Berlin Springer Verlag. p. 203 (arXiv:0909.2579), doi:10.1007/978-3-540-76937-8_8

McHardy I., Uttley P., Taylor R., Papadakis I., 2006, in Gaskell C. M., McHardy I. M., Peterson B. M., Sergeev S. G., eds, Astronomical Society of the Pacific Conference Series Vol. 360, Astronomical Society of the Pacific Conference Series. p. 85

Nandra K., George I. M., Mushotzky R. F., Turner T. J., Yaqoob T., 1997, ApJ, 476, 70

O’Neill P. M., Nandra K., Papadakis I. E., Turner T. J., 2005, MNRAS, 358, 1405

Osterbrock D. E., Pogge R. W., 1985, ApJ, 297, 166

Padovani P., Giommi P., 1995, ApJ, 444, 567

Paliya V. S., Böttcher M., Diltz C., Stalin C. S., Sahayanathan S., Ravikumar C. D., 2015, ApJ, 811, 143

Pancoast A., Brewer B. J., Treu T., 2014, MNRAS, 445, 3055

Papadakis I. E., McHardy I. M., 1995, MNRAS, 273, 923

Peterson B. M., et al., 2000, ApJ, 542, 161

Petrucci P. O., et al., 2000, ApJ, 540, 131

Petrucci P.-O., et al., 2013, A\&A, 549, A73

Pian E., et al., 1998, ApJ, 492, L17

Ponti G., Papadakis I., Bianchi S., Guainazzi M., Matt G., Uttley P., Bonilla N. F., 2012, A\&A, 542, A83

Pounds K. A., Done C., Osborne J. P., 1995, MNRAS, 277, L5

Press W. H., Teukolsky S. A., Vetterling W. T., Flannery B. P., 1992, Numerical recipes in C. The art of scientific computing

Pryal M., Falcone A., Stroh M., 2015, ApJ, 802, 33

Ravasio M., Tagliaferri G., Ghisellini G., Tavecchio F., Böttcher M., Sikora M., 2003, A\&A, 408, 479

Ravasio M., Tagliaferri G., Ghisellini G., Tavecchio F., 2004, A\&A, 424, 841

Rees M. J., 1984, ARA\&A, 22, 471

Reis R. C., et al., 2012, ApJ, 745, 93

Romero G. E., Cellone S. A., Combi J. A., 1999, A\&AS, 135, 477

Sembay S., Warwick R. S., Urry C. M., Sokoloski J., George I. M., Makino F., Ohashi T., Tashiro M., 1993, ApJ, 404, 112

Sikora M., Begelman M. C., Rees M. J., 1994, ApJ, 421, 153

Sobolewska M. A., Papadakis I. E., 2009, MNRAS, 399, 1597

Soldi S., et al., 2008, A\&A, 486, 411

Soldi S., et al., 2014, A\&A, 563, A57

Tagliaferri G., et al., 2000, A\&A, 354, 431

Takahashi T., et al., 1996, ApJ, 470, L89

Tanihata C., et al., 2000, ApJ, 543, 124

Tanihata C., Takahashi T., Kataoka J., Madejski G. M., 2003, ApJ, 584, 153

Turner T. J., Miller L., 2009, A\&ARv, 17, 47

Turner T. J., George I. M., Nandra K., Turcan D., 1999, ApJ, 524,667

Ulrich M.-H., Maraschi L., Urry C. M., 1997, ARA\&A, 35, 445

Urry C. M., Padovani P., 1995, PASP, 107, 803

Uttley P., McHardy I. M., Papadakis I. E., 2002, MNRAS, 332, 231

Vaughan S., Edelson R., Warwick R. S., Uttley P., 2003, MNRAS, 345,1271

Véron-Cetty M.-P., Véron P., 2010, A\&A, 518, A10 
Wagner S. J., Witzel A., 1995, ARA\&A, 33, 163

Wierzcholska A., Wagner S. J., 2016, MNRAS, 458, 56

Zhang Y. H., et al., 2002, ApJ, 572, 762

Zhang Y. H., Treves A., Celotti A., Qin Y. P., Bai J. M., 2005, ApJ, 629, 686

Zhang Y. H., Treves A., Maraschi L., Bai J. M., Liu F. K., 2006, ApJ, 637, 699

Zu Y., Kochanek C. S., Peterson B. M., 2011, ApJ, 735, 80

Zu Y., Kochanek C. S., Kozłowski S., Udalski A., 2013, ApJ, 765, 106 